\title{
Hypoxic Behavior in Cells under Controlled Microfluidic Environment
}

\author{
Adnan Morshed and Prashanta Dutta§ \\ School of Mechanical and Materials Engineering \\ Washington State University, Pullman, WA 99164-2920
}

Tel: (509) 335-7989; Fax: (509) 335-4662; Email: prashanta@wsu.edu

$\S$ Corresponding Author

(C) 2017. This manuscript version is made available under the Elsevier user license

http://www.elsevier.com/open-access/userlicense/1.0/ 


\begin{abstract}

\section{Background}

Depleted oxygen levels, known as hypoxia, causes considerable changes in the cellular metabolism. Hypoxia-inducible factors (HIF) act as the major protagonist in orchestrating manifold hypoxic responses by escaping cellular degradation mechanisms. These complex and dynamic intracellular responses are significantly dependent on the extracellular environment. In this study, we present a detailed model of a hypoxic cellular microenvironment in a microfluidic setting involving HIF hydroxylation.

\section{Methods}

We have modeled the induction of hypoxia in a microfluidic chip by an unsteady permeation of oxygen from the microchannel through a porous polydimethylsiloxane channel wall. Extracellular and intracellular interactions were modeled with two different mathematical descriptions. Intracellular space is directly coupled to the extracellular environment through uptake and consumption of oxygen and ascorbate similar to cells in vivo.
\end{abstract}

\title{
Results
}

Our results indicate a sharp switch in HIF hydroxylation behavior with changing prolyl hydroxylase levels from 0.1 to $4.0 \mu \mathrm{M}$. Furthermore, we studied the effects of extracellular ascorbate concentration, using a new model, to predict its accumulation inside the cell over a relevant physiological range. In different hypoxic conditions, the cellular environment showed a significant dependence on oxygen levels in resulting intracellular response.

\section{Conclusions}

Change in hydroxylation behavior and nutrient supplementation can have significant potential in designing novel therapeutic interventions in cancer and ischemia/reperfusion injuries.

\section{General significance}

The hybrid mathematical model can effectively predict intracellular behavior due to external influences providing valuable directions in designing future experiments. 


\section{Introduction}

Rapid tumor growth can result in localized zones in the tumor microenvironment where cells have far less access to nutrients. It has often been observed that in these zones cells get very little oxygen due to the inadequate or defective vasculature, a condition known as hypoxia [1]. The irregularities in functional behavior as well as structure of the blood vessels in solid tumors have been identified as one of the main reason for oxygen deficiency in the tissue [2]. Hypoxia is also known to be induced in heart and brain cells through ischemia [3]. In some instances, these zones might have no access to oxygen supply at all (a condition known as anoxia) which could lead to cell apoptosis and necrosis [4]. Although it is known that healthy cells can be severely damaged in hypoxia, cancer cells show significant resistance in hypoxic and anoxic conditions [5]. Moreover, hypoxic cancer cells have shown decreased susceptibility to radiotherapy and chemotherapy [6]. It is, therefore, a topic of interest from the therapeutic point of view to study the cell behavior in hypoxia.

Hypoxia-inducible factors (HIF) are a family of transcription factors that are highly active in cells under conditions of low levels of oxygen [7]. In normoxic conditions, isoforms of the prolyl hydroxylase (PHD) domains in the cell cytoplasm bind with HIF and act as the HIF regulator [8] (see Figure 1). This regulation, however, is dependent on the kinetic activation (or catalysis) of PHD by a number of other nutrients [9]. In hypoxic and anoxic conditions, the lack of oxygen impedes the hydroxylation of HIF. This causes an accumulation of HIF1 $\alpha$, a prominent HIF isoform, in the cytoplasm which shuttles into the nucleus [10] and binds with HIF1 $\beta$ to form a dimer complex $\left(\mathrm{HIF}_{\mathrm{d}}\right)$ [11]. This HIF dimer transcriptionally activates large number genes at the hypoxia response element (HRE) sites. Significant target genes include those controlling erythropoiesis and iron metabolism [12]; influencing proliferation like insulin growth factors (IGF) and transforming growth factors (TGF) [13]; regulating vascular tone and angiogenesis such as vascular endothelial growth factor (VEGF) [14]; controlling energy metabolism like aldolase, enolase, pyruvate kinase and glucose transporters [15]. All these factors have made the cellular dynamics of HIF a topic of prime interest in the study of ischemia and tumor growth [16]. 

in human cells in vivo, it is still not technologically or logistically feasible. A large number of in vivo mouse xenograft models [17] and in vitro studies using different cell lines have clearly shown the accumulation of HIF1 $\alpha$ in the cell and the associated transcriptional activities [15]. Microfluidic cell culture devices have been used in recent years as in vitro platforms where it is possible to precisely maintain viable microenvironments (both temporally and spatially) for studying cellular pathways [18]. For instance, microfluidic devices have been used to study cancer cell growth, cell cycle, apoptosis, and other mechanisms [19]. These low-cost devices can particularly be used to study multiple cell lines under exactly same nutritional and pathological conditions providing better integration of operations and an efficient way to study the heterogeneity of cell populations [20, 21]. Moreover, these devices can be used for anticancer drug testing and to study the cellular response over desired time span under easily reproducible conditions, which are not possible in traditional in vitro experiments [22]. More importantly, the microenvironment in these microfluidic cell culture devices can be used to closely mimic many in vivo phenomena like the transition to invasive phenotype of the cells in the tumor microenvironment $[23,24]$.

Study of cellular response to oxygen starvation and nutrient supplementation in the microfluidic environment necessitates simultaneous spatial and temporal measurements of a large number of species like different forms of prolyl hydroxylase domains, hypoxiainducible factors, ascorbate, oxygen, iron etc., which presents a significant challenge in the currently available optical measurement techniques. Moreover, microenvironmentdependent extracellular to intracellular and cytosolic to nuclear shuttling of the nutrients play a crucial role in determining the outcome of the cellular dynamics. However, it is extremely difficult to measure concentrations of the same species in different cellular compartments like the nucleus, mitochondria and the cytoplasm in vitro. Mathematical models are of significant benefit in this regard, where it is possible to investigate selected pathways in greater detail than it is possible with experimental methods. Coupled with experimental studies, mathematical models of the cellular microenvironment can provide meaningful insight into therapeutic studies. More importantly, it can venture beyond the 
logistical limitations of experimental assays and provide directions for specific experimental studies, thus saving resources and time.

A number of mathematical models have been presented with different levels of detail for the intracellular reactions in hypoxic condition. Some of these models investigated pathways involving oxygen sensing mechanism by HIF1 $\alpha$ and the roles of different intracellular components on HIF1 $\alpha$ hydroxylation [25], while others focused into cytosolic to nuclear shuttling of nutrients and HIF1 $\alpha$ at different stages of the HIF1 $\alpha$ hydroxylation as well as post-hypoxic activities in a single cell model [26]. All these models, however, consider only the intracellular dynamics and for the most part, treat the species inside as a well-mixed system, resulting in a set of ordinary differential equations representing the cellular dynamics. However, it is well established that the microenvironment appreciably influences cellular metabolism especially when the supply of nutrients are being impeded externally [27]. It is also known that cellular uptake and consumption of nutrients like oxygen, ascorbate and iron from the extracellular environment are continuous processes, which cannot be properly represented only with an intracellular mathematical model. Additionally, the available cell-based intracellular models are not adequate to provide a satisfactory description of the microfluidic cell culture device, where nutrients are supplied in the extracellular domain, and the hypoxic environment is also maintained using extracellular control.

In this study, we have presented a hybrid model to study the transport and evolution of different species in both extracellular and intracellular spaces. Changes in the upstream conditions, such as nutrient supplementation and oxygen extraction in the extracellular domain, have been incorporated to represent the actual microfluidic experiments. We have developed a reduced order mathematical representation of the physical microfluidic cell culture environment to investigate the cellular behavior under hypoxic and normoxic conditions. Parameters dictating the complex permeation process have been identified for the reduced model and a comparison with the existing experimental data is presented. We have also developed a generalized cellular ascorbate uptake model validated by experimental findings that connect the extracellular ascorbate levels to the intracellular one. 


\subsection{Extracellular Space}


In this microfluidic system, the extracellular medium consists of water and nutrients, such as oxygen, ascorbate, and iron. The transport of the nutrients in the extracellular space is modeled with the species mass conservation equation [33],

$$
\frac{\partial C_{i}}{\partial t}+\nabla \cdot\left(\bar{u} C_{i}\right)=\nabla \cdot\left(D_{i} \nabla C_{i}\right)+R_{i}
$$

where $C_{i}$ is the instantaneous spatial concentration of nutrient species $i, \bar{u}$ is the medium fluid velocity, $D_{i}$ is the diffusion coefficient for species $i$ in the medium, and $R_{i}$ is the synthesis or degradation of species $i$ per unit volume due to chemical reactions. The necessary initial and boundary conditions for each species are provided in table 1.

In hypoxic chips, dissolved oxygen is forced to leave the microfluidic cell culture chamber by permeation through the top PDMS layer, as shown in Figure 2(b), due to the concentration gradient across PDMS. Here, the driving mechanism for the oxygen efflux is the difference in the partial pressure of oxygen across the membrane. Due to this difference, dissolved oxygen from the liquid mixture comes out in the gas phase and diffuses through the porous PDMS layer [30]. This permeation process is sensitive to PDMS membrane thickness, temperature, the flow velocity of the medium inside the channel, and more importantly, flow velocity of the gas phase outside the channel. Detailed model for these physical phenomena has been presented as a two-dimensional diffusion problem with discontinuous solubility across the membrane [34]. More recently, Brennan et. al. presented this transport process in a simplified model from a pressure dependent desorption kinetics approach [29].

In this study, we have presented a novel reduced order model considering the disparate length scale in our microfluidic device. Since the channel length $(3 \sim 5 \mathrm{~cm})$ is much higher than the height (10 to 100 microns), the variation of concentration along the smaller dimensions is much smaller compared to that along the length. This limiting simplification holds for the present case where the height-wise variations are negligible compared to the length-wise variations. Although this simplifies Eq. (1) considerably, care must be taken when transport through boundary walls is present or a significant heightwise concentration gradient is created. For our case due to the difference in bulk 
concentration of oxygen inside the channel and outside environment (artificially maintained; e.g. inside hypoxia chamber [28]) we see an efflux of oxygen through the oxygen permeable PDMS layer. It must be stressed that this permeation is directly proportional to the difference in concentration across the PDMS layer [29]. Thus, using the analogy of heat transfer, the problem can be represented mathematically as a quasi- twodimensional transport with boundary contributions as localized source/sink terms. In this case, the flux of oxygen across the PDMS layer can be simplified to,

$$
O_{\text {efflux }}=P_{c}\left(C_{O, \text { inside }}-C_{O, \text { outside }}\right)
$$

where $P_{c}$ is called the permeation constant [35]. Phenomenologically, this transport will increase with increasing channel surface area through which the permeation takes place. In this formulation it is assumed that the concentration variation along the channel height is negligible, which can be justified for very thin microchannel problem considered here [29]. Hence, the governing equation for oxygen transport can be given as,

$$
\frac{\partial C_{O}}{\partial t}+\nabla \cdot\left(\bar{u} C_{O}\right)=\nabla \cdot\left(D_{O} \nabla C_{O}\right)+R_{O}-P_{c}\left(C_{O, \text { inside })}-C_{O, \text { outside }}\right)
$$

where the second term on the right-hand side represents generalized reactions and uptake in the domain.

Similar to oxygen, the transport of ascorbate through the microchannel is modeled as

$$
\frac{\partial C_{A S}}{\partial t}+\nabla \cdot\left(\bar{u} C_{A S}\right)=\nabla \cdot\left(D_{A S} \nabla C_{A S}\right)-R_{A S}
$$

where $R_{A S}$ is the sink term for ascorbate uptake by the cells described in detail in the following section.

\subsection{Interaction between Extracellular and Intracellular Regions}

\subsubsection{Oxygen Uptake}

Cells consume oxygen from the extracellular region to meet the energy production and other metabolism requirements. Most of this oxygen is used by mitochondria for energy 
production [36]. In tumor cells, although the onset of hypoxia significantly reduce oxygen usage by mitochondria, HIF increases anaerobic glycolysis which still provides the tumor cells with energy to survive in the hypoxic environment [37].

In this study, we model the consumption of oxygen by the cell as a reactive sink term localized at the cell boundary

$$
R_{O}=-\rho_{\text {cell }} V_{\max , O} \frac{\left[C_{O}\right]}{k_{m, O}+\left[C_{O}\right]}
$$

where $\rho_{\text {cell }}$ is the volumetric cell concentration and $C_{O}$ is the extracellular concentration of oxygen. The parameter $V_{\max , O}$ can be obtained from experimental measurements of cellular oxygen uptake in air-saturated medium, and it varies depending on the type of cell in question [38]. On the other hand, a number of experimental measurements suggested that the constant $k_{m, O}$ is on the order of $1 \mu \mathrm{M}$ or less for cellular oxygen uptake [38]. The parameter values used in this study are provided in table 2 .

\subsubsection{Ascorbate Uptake}

Vitamin C (aka ascorbate) gets into the cell through various oxidized states, such as ascorbic acid, ascorbate, ascorbate free radical and dehydroascorbic acid (DHA) [39]. While ascorbate is accumulated in cells by sodium-dependent vitamin C transporters (SVCTs), DHA is absorbed via sodium-independent facilitative glucose transporters (GLUTs) followed by intracellular reduction [40]. Amount and availability of these transporters vary in different kinds of cells. The selectivity of these transporters to different forms of ascorbate also differs widely. Although the uptake of intestinal ascorbate is very tightly regulated [41], recent pharmacokinetic data suggests that intravenous administration of ascorbate can result in much higher ascorbate level in plasma [42]. Except for human erythrocytes, intracellular ascorbate concentrations have consistently been found in higher concentrations than extracellular fluids [39]. However, the uptake rate has been found as a function of extracellular concentrations and time, suggesting some form of enzymesubstrate kinetics dictating the uptake process [43]. Although the experimental data from [44] suggests different uptake rates for different extracellular concentrations, the rates 
remain steady over the initial five hours. Hence, we constructed an extracellular concentration dependent uptake model for ascorbate that follows Michaelis-Menten kinetics.

$$
R_{A S}=\rho_{\text {cell }} A S_{\text {in }}=\rho_{\text {cell }} V_{\operatorname{mxx}, A S} \frac{\left[A S_{\text {ext }}\right]}{k_{m, A S}+\left[A S_{\text {ext }}\right]}
$$

Here $A S_{\text {in }}$ and $A S_{\text {ext }}$ denote the rate of ascorbate uptake and the extracellular ascorbate concentration, respectively. Since the ascorbate transport is transporter protein- (SVCT1, SVCT2, Glut etc.) mediated, the uptake dynamics will change based on the cell types (more specifically, depend on the activity of the different transporter proteins in that specific type of cell) under study and the parameters should be adjusted accordingly. Here, we have generalized the uptake process based on the experimental results presented in [44], without being specific to any given transporter protein. Rather, this model can be adapted for any cell lines irrespective of the transport mechanisms involved, if sufficient experimental data is available to adjust the Michaelis-Menten parameters. Equation (6) is later included in the intracellular evolution of ascorbate as the uptake contribution (see Eq. (S1.8)). The estimated parameter values for ascorbate uptake are also reported in table 2.

\subsection{Intracellular Dynamics and Pathways}

The intracellular biochemistry is complex and it depends on both internal and external environments. It has been reported that the onset of hypoxia brings about a large number of gene activations, which brings change in the intracellular equilibrium [45]. Since gene activations happen inside the nucleus, in our model, we compartmentalize the cell into two sections corresponding to the cytoplasm and the nucleus. While reactions in the cytoplasm continue in all conditions, the hypoxic reaction pathways are enabled once the cellular oxygen level reaches down to hypoxic levels. The biochemical models for normoxia and hypoxia are simplified to some extent in order to facilitate analysis. Nevertheless, they retain all the essential characteristics of the pathways as shown in Fig. 1.

\subsubsection{Pathway for Normoxia}


The intracellular HIF $1 \alpha$ is hydroxylated (HIF1 $\alpha \mathrm{h}$ ) by PHD in the presence of oxygen $\left(\mathrm{O}_{2}\right)$ and other co-reactors such as iron (Fe), 2-oxoglutarate (DG), ascorbate (AS), etc. as

$$
\begin{gathered}
P H D+F e \rightleftharpoons P F \\
P F+D G \rightleftharpoons P F D \\
P F D+O_{2} \rightleftharpoons P F D O \\
P F D O+A S \rightleftharpoons P F D O A
\end{gathered}
$$

Though different PHD isoforms perform different activities and have specific nuclear or cytoplasmic localization [46], for simplicity all of these isoforms are treated as one component in the pathway. However, the cytosolic to nuclear shuttling of activated PHD is considered in the model. The binding of PHD with iron, 2-oxoglutarate, oxygen [47] and ascorbate was assumed bi-directional with negligible intermediates [48]. However, the reversible binding of the PHD complex with HIF1 $\alpha$ produces a PFDOA-HIF1 $\alpha$ intermediate. This intermediate then proceeds to produce the hydroxylated HIF1 $\alpha$. The sequential binding of collagen prolyl hydroxylase during hydroxylation has been identified to proceed in the order of iron $\rightarrow 2$-oxoglutarate $\rightarrow$ oxygen $\rightarrow$ peptide [49]. The same sequence of binding has been suggested for HIF hydroxylation by PHD [50]. In normoxia, this primarily unidirectional hydroxylation reaction ensures a steady drop in intracellular HIF1 $\alpha$ concentration.

$$
P F D O A+H I F 1 \alpha \rightarrow \text { HIF1 } \alpha \mathrm{h}
$$

Following the hydroxylation, HIF1 $\alpha$ h becomes available for binding with vHL ubiquitin ligase complex. In normoxia, an abundance of vHL in the cytoplasm ensures the irreversible proteasomal destruction of HIF1 $\alpha$ h, causing the steady degradation of HIF1 $\alpha$ h levels inside the cell [51].

$$
(\mathrm{HIF} 1 \alpha \mathrm{h})+\mathrm{vHL} \rightarrow \text { Degradation }
$$

\subsubsection{Pathway for Hypoxia}

During hypoxia, HIF1 $\alpha$ shuttles inside the nucleus where it binds with HIF1 $\beta$ forming the $\mathrm{HIF}_{\mathrm{d}}$ dimer $[10,45]$.

$$
\mathrm{HIF} 1 \alpha \rightarrow \operatorname{HIF} 1 \alpha_{\mathrm{n}}
$$




$$
\mathrm{HIF} 1 \alpha_{\mathrm{n}}+\mathrm{HIF} 1 \beta \rightleftharpoons \mathrm{HIF}_{d}
$$

This dimer acts as the key proponent in gene activation and a host of transcriptional activities as

$$
\begin{gathered}
\mathrm{HIF}_{d}+\mathrm{HRE} \rightleftharpoons\left(\mathrm{HIF}_{d} \mathrm{HRE}\right) \\
\left(\mathrm{HIF}_{d} \mathrm{HRE}\right) \rightarrow \text { Transcriptional Activity }
\end{gathered}
$$

As mentioned in the previous section, activated PHD may shuttle in the nucleus from the cytosol and can hydroxylate nuclear HIF1 $\alpha$ [26].

$$
\begin{aligned}
& \mathrm{PFDOA} \rightleftharpoons \mathrm{PFDOA}_{\mathrm{n}} \\
& \text { HIF } 1 \alpha_{n}+\text { PFDOA }_{n} \rightarrow{\text { HIF } 1 \alpha_{n} h}
\end{aligned}
$$

Finally, the nuclear HIF1 $\alpha$ may also undergo vHL induced proteasomal degradation [45].

$$
\left(\operatorname{HIF} 1 \alpha_{\mathrm{n}} \mathrm{h}\right)+\mathrm{vHL} \rightarrow \text { Degradation }
$$

The reactions (7 to 12) represent the normoxic pathway, while the reactions (7 to 19) are representative of the hypoxic response. In order to mathematically represent these chemical reactions, ordinary differential equations are formulated following mass-law kinetics as shown in table S1. For example, the temporal evolution of intracellular ascorbate (Eq S1.8) involves binding with PFDO complex resulting in a reduction proportional to their instantaneous concentrations (first term), a regain proportional to the amount available through the breakdown of the PFDOA complex (second term), and overall uptake from extracellular region constituting a rise (third term).

\section{Numerical Methods}

A hybrid numerical algorithm is developed in this study to represent the cellular dynamics in a microfluidic environment. While the intracellular interactions require solving a set of ordinary differential equations (Eqs. S1.1-S1.22) under the assumption of a well-mixed system inside the cell, cellular compartmentalization is also maintained to separate cytosolic and nuclear interactions by introducing compartment-specific species. Intercompartmental shuttling is included in the equations to represent communication between the compartments. The extracellular environment, on the other hand, is a multi- 

each of the extracellular species (Eq. 1). Cellular boundaries are considered to be aligned with domain grids, while cellular consumption (Eqs. 5 and 6) of nutrients from extracellular space is considered as a link between the extracellular and intracellular domains.

The governing equations for the extracellular space are solved numerically using finite volume technique [52, 53]. The transport equations (Eqs. 3 and 4) for nutrient species are discretized following exact (exponential) formulation [54], while the temporal evolution is resolved as first order implicit scheme. An in-house code was developed in FORTRAN 95 for the solution of the system. Discretized algebraic equations are solved iteratively using tridiagonal matrix algorithm (TDMA) to find oxygen and ascorbate concentration. For iterative scheme, we used a convergence tolerance of $10^{-5}$ until proceeding to the next time step. Grid refinement was done until results were meshindependent. The time step for the temporal evolution was set as 0.05 seconds and the spatial grid was refined to $10.0 \mu \mathrm{m}$ by $3.0 \mu \mathrm{m}$. While solving the species transport equations, physical regions containing the cells were numerically blocked off by modifying the relevant transport properties such as viscosity and diffusion coefficients (please see Ref [55] for details). These regions are studied in detail through a set of ordinary differential equations providing the temporal evolution of all the species inside the cells. The contributions of source/sink terms from Eqs. 5 and 6 are localized only in the control volumes adjacent to the biological cells.

For intracellular components, a fourth order Runge-Kutta (RK) algorithm is used to solve a set of ordinary differential equations (Eqs. S1.1 - S1.22). Necessary reaction constants and initial conditions (concentrations) for these equations are used from values reported in the literature as listed in table S2 and S3. Figure 3 illustrates the overall solution algorithm used in this study for both extracellular and intracellular regions. The numerical calculations were carried out on a Linux platform using a $2.4 \mathrm{GHz}$ Intel Xeon CPU. 


\section{Results and Discussion}

We examined detailed cellular behavior in the microchannel for different levels of oxygen supply. The most common representation of oxygen level is the volume fraction of oxygen in the air supplied to the cell medium. The actual concentration of oxygen can then be calculated from its solubility. Thus, for an oxygen solubility $1.30 \mu \mathrm{mol} / \mathrm{l} / \mathrm{mmHG}$ in water (at $37^{\circ} \mathrm{C}$ and standard pressure), $21 \%$ ambient oxygen corresponds to $207 \mu \mathrm{M}$ oxygen in the solution [56]. Inside the cell culture device, the spatial distribution of oxygen is calculated by solving Eq. 3 at each time step. Figure 4 shows the temporal evolution of oxygen concentration in the microfluidic cell culture device (Fig. 2(b)). Numerical results are compared with identical experimental observation [28], where a chip containing human prostate cancer cell was placed inside a hypoxia chamber. As the oxygen in the hypoxia chamber was displaced by nitrogen flushing, oxygen from the cell culture chip started to permeate out resulting in the desired hypoxic conditions. As noted before, in our numerical model, the oxygen permeation is taken care by a sink term in Eq. 3, which is controlled by the difference of oxygen levels across the PDMS layer. The initial concentration of oxygen inside the microchannel is considered at $21 \%$, while the oxygen concentration was set at $0 \%$ on the top boundary (PDMS layer) of the microchannel resulting from nitrogen flushing. During the onset of the hypoxic condition, there was no bulk flow in the cell culture chamber. Thus, the fluid inside the microfluidic chip was considered static, and the diffusion coefficient for oxygen in the aqueous medium was taken as $2.66 \times 10^{-9} \mathrm{~m}^{2} / \mathrm{s}$ [57]. Our numerical results show good agreement with experimental data (red squares) for a permeation constant of $2.35 \times 10^{-2} \mathrm{~s}^{-1}$.

Next, we present the intracellular dynamics of HIF1 $\alpha$ under normoxic (21\% oxygen) and hypoxic (1\% oxygen) conditions. The initial concentrations of species outside the nucleus were chosen following previous models $[25,58]$ which were originally based on the in vitro experiments carried out by Tuckerman et al. [56]. The actual in vivo tissue concentrations of oxygen are significantly lower in normoxia (6-25 $\mu \mathrm{M})$ [25]. This kind of standard cell culture conditions is therefore 'hyperoxic' compared to cellular physiological levels [59]. Hence, it is not uncommon to see HIF levels overestimated in numerical models (which is in nanomolar range in normal cellular conditions), and consequently a higher 
concentration for PHD to maintain a prescribed PHD to HIF ratio [58]. On the other hand, model concentrations for nuclear species (e.g. HIF1 $\beta$ ) were chosen from values reported in the work by Nguyen et al. [26]. In figure 5(a), we have compared model results of maximal vHL protein capture in normoxia and hypoxia with experimental data from Tuckerman et.al. [56]. In their experiments, they have estimated the activity of endogenous HIF-PHDs in-vitro by a vHL capture assay. Here we have maintained the exact reaction parameters following the experimental data for model validation where the initial PHD concentration is $4 \mathrm{nM}$ [56]. Given the adequate presence of required co-reactors such as 2-oxoglutarate, iron, and ascorbate, normoxic oxygen levels produce faster hydroxylation than hypoxic levels, and consequently more hydroxylated PHD-HIF complexes are available in normoxia to bind with vHL protein than hypoxic scenarios. Compared to experimental measurements [56], the numerical results overestimate the maximum vHL capture in hypoxia after 5 minutes. This could result from the model choice of cytosolic to nuclear transport of PHD only after it has been bound to 2-oxoglutarate, iron, oxygen and ascorbate (figure 1, steps 1-4). However, some other models [26] have suggested that the PHD might be shuttling in the nucleus during each step of its binding to different co-reactors, which would result in a lower level of PFDOA after step 4 and consequently lower than $20 \%$ of maximal pvHL capture after 10 minutes at $1 \%$ oxygen.

The relative quantity of HIF $1 \alpha$ during hypoxia from $1 \%$ oxygen to $6 \%$ oxygen range is presented in figure 5(b). Here, for a given duration of hypoxia (10, 20 or 60 minutes), the results are normalized to the level of unhydroxylated HIF1 $\alpha$ present at $6 \%$ oxygen. Like Qutub and Popel [25], the HIF that is not hydroxylated after the given duration of hypoxia is considered as the accumulation. The numerical results (blue triangles) after 20 minutes of hypoxia from [25] as well as experimental results (red squares) from [51] are included in the figure. As the oxygen level declines below 6\%, the amount of unhdroxylated HIF $1 \alpha$ increases significantly. This amount is also dependent on the hypoxia exposure time. Holding at a given hypoxic condition longer allows the HIF $1 \alpha$ to bind with HIF1 $\beta$ inside the nucleus which results in the drop in free HIF1 $\alpha$ level. At the same time, some of it is also hydroxylated inside the nucleus. Our results completely capture the HIF dynamics when compared to the experimental measurements done in hypoxia (from $1 \%$ to $6 \%$ oxygen). 
However, ours as well as the numerical results presented in [25] deviated from the experimental values beyond 1\% oxygen level. This deviation is most likely due to the added production of HIF $1 \alpha$ in the nucleus once the hypoxia reaches this kind of chronic levels, which is not included in the present model. The present model primarily focused in the hypoxic region between $1 \%$ and $6 \%$ where the agreement is excellent. Further validation of this model is given in terms of the HIF half-life in the supplementary materials. It should be noted that due to the lack of microfluidic experimental data, the intracellular validations are primarily based on different in-vitro experiments. Different studies used different experimental conditions, cell types and methods. However the general intracellular dynamics used in the model is representative of the primary processes, and variational studies presented in the following discussions provide a way for observing the change in the dynamics with different levels of intracellular concentrations of the key species.

The temporal evolution of HIF1 $\alpha$ in hypoxic conditions can be envisaged in further detail with its reallocation in the nucleus. In figure 6(a) we present the cytosolic HIF content over a period of one hour under given conditions of $1 \%$ and $6 \%$ oxygen, while figure 6(b) shows the translocated HIF1 $\alpha$ in the nucleus under the same conditions. Lower oxygen levels mean more HIF1 $\alpha$ is available for nuclear shuttling as shown in figures 6(a) and 6(b) for a time period of one hour. The initial sharp jump in figure 6(b) is due to the assumption of negligible nuclear HIF1 $\alpha$ at the start of the shuttling process. Inside the nucleus, incoming HIF $1 \alpha$ is immediately engaged by both HIF1 $\beta$ and PFDOA. These two parallel reactions produce the HIF dimer and hydroxylated HIF $1 \alpha$, respectively and consequently, result in very little deposition of free HIF1 $\alpha$ inside the nucleus as shown in figure 6(b). The general nuclear response in the hypoxic conditions is presented in figure 6(c). The responses presented here are the normalized transcriptional activity (TA) resulting from mRNA activation by hypoxia response elements inside the nucleus as depicted in figure 1 . Since the activity was highest for the oxygen level of $1 \%$, its value at the end of one hour was used to normalize the response. The prolonged hypoxic scenario presented here is physically very similar to a rapid proliferation stage of a ductal carcinoma in-situ (DCIS), where the lack of adequate vasculature in growing tumor cell colony often leads to severe hypoxic and even anoxic conditions at the core of the solid 
mass [37]. Although a necrotic core can result from such dearth of nutrients in ductal carcinoma, a vast number of experimental investigations have shown the shift in cellular metabolism to adapt to low oxygen and nutrient levels, and in some cases a switch to a dormant stage to ensure survival in tumor cells. These metabolic effects, as well as a number of pleiotropic response to hypoxic stress, have been shown to be mediated by a number of regulatory genes (for example, glucose transporters, angiogenic growth factors, hexokinase and hematopoietic factors) which are activated through hypoxia response inside the nucleus [60]. This kind of transcriptional activity can be directly correlated to the hypoxic response element activity presented in figure 6(c).

It should be noted that, in the cytoplasm, as long as some oxygen is available, the proteasomal degradation of HIF1 $\alpha$ continues, albeit at significantly reduced quantity in hypoxic conditions. The small amount of HIF1 $\alpha$ hydroxylated in these scenarios (figure 6(d)), are immediately available for vHL protein-mediated degradation, which prevents any HIF1 $\alpha$ h stabilization. On the other hand, any mutation in vHL protein affects its functionality. For instance, in renal carcinoma, the degradation mechanism of vHL protein is inhibited, and in this case hydroxylated HIF1 $\alpha$ is deposited in the cytoplasm (figure 6(d), dotted lines) [61]. A number of experimental and clinical studies have attributed the vHL protein inhibition to somatic mutations [62] and mutations in the non-coding regions of vHL [63]. Our results indicate that a significant four orders of magnitude higher accumulation of hydroxylated HIF1 $\alpha$ when the vHL mediated degradation was turned off. Considering this degradation mechanism functional, it is reasonable to assume in our model that the hydroxylation of HIF $1 \alpha$ is unidirectional given the minute quantity of HIF1 $\alpha$ h left (Fig. 6d). However, as our results suggest, for a defective vHL degradation condition, this assumption should be reconsidered and the reverse dehydroxylation reaction should also be included.

Hypoxic behavior with varying levels of PHD are presented in figures 7(a) and 7(b) for 1\% oxygen case. The changes in PHD levels in hypoxia (from nanomolar to micromolar range) follows the argument presented in [25] that the over-expression of PHD results due to the cellular synthesis of PHD proteins (along with HIF1 $\alpha$ ) when the cells are exposed to more than 3 hours of hypoxia. It should also be noted that we lumped PHD1, PHD2 and 

(PHD2 minimally; PHD3 strongly [64]) has already been observed experimentally, especially during longer exposures $[59,65]$. Moreover, different cancer cell lines exhibit different PHD:HIF ratios and a number of them are still to be quantified [25]. Based on the relative amount of HIF and PHD in the system, the hydroxylation dynamics change even though the other cofactors are maintained at the same level. Conversely, to maintain the same level of hydroxylation across different cell lines, the relative amount of PHD must be varied which was shown previously in a different model with experimental validation [58]. Hence, it is useful to investigate the HIF dynamics over a broad range.

While oxygen concentration is the most ubiquitous factor controlling PHD-catalyzed hydroxylation reactions, all PHD isoforms employ 2-oxoglutarate (DG) as co-substrates, and iron and ascorbate as cofactors [66]. Therefore, selective inhibition of these cofactors can have a significant effect on cellular free PHD levels. Although fully functional PHD pathway results in HIF destabilization and degradation under normoxic conditions, in hypoxia, when HIF is stabilized, it can transcriptionally produce PHD2 and PHD3 isoforms resulting a negative feedback mechanism for an increased PHD concentration. From the perspective of cancer therapy, increasing PHD has the potential to arrest hypoxic activity appreciably causing a switch-like drop in HIF and HIF-mediated transcriptional activity as shown in figure $7(a, b)$ for the limiting case of $4 \mu \mathrm{M}$ PHD.

However, certain other therapeutic scenarios require PHD reduction/inhibition as well. For example, anemia, myocardial infarction, and stroke are directly associated with hypoxia in the tissue level. HIF stabilization through PHD pathway inhibition in these settings can result in the higher HIF-mediated production of red blood cell, augmented glycolysis and mitochondrial autophagy, vasodilation and angiogenesis [67, 68]. PHD inhibition or a lower expression of PHD will produce a markedly higher hypoxia response (see Figure 7(a, b) for 1.0 and $100 \mathrm{nM}$ PHD), an effect already being employed to increase tissue survival in preclinical models of ischemic renal disease [69], myocardial infarction, and stroke $[70,71]$. Our results show very little change in hypoxic response when PHD levels are reduced 100 fold from $0.1 \mu \mathrm{M}$. On the contrary, a drastic drop in transcriptional activity is achieved when PHD is increased just one order from the same $0.1 \mu \mathrm{M}$ level 
revealing a transitional effect on HIF dynamics between $0.1 \mu \mathrm{M}$ and $4.0 \mu \mathrm{M}$ of PHD concentration.

The presence of succinate poses an impediment in PHD binding of 2-oxoglutarate through competitive binding [72]. A number of studies have related excess production of succinate with mitochondrial complex II malfunction along with lethal amounts of reactive oxygen species [73], which incidentally is retarded by HIF in many cases in a regulatory fashion to ensure cell survival. Higher succinate levels thus have the ability to produce similar therapeutic effects in clinical cases requiring HIF stabilization as shown in Figure 7(d). Here the transcriptional activities are normalized with the level present after one hour for $1.0 \mathrm{nM}$ PHD (figure 7(b)). Overall, the change in HIF activity and hypoxia response (figure $7(\mathrm{c}), 7(\mathrm{~d})$ ) remain gradual with decreasing 2-oxoglutarate levels. Here the upper limit of the 2-oxoglutarate range was chosen following the in vitro parameters of Tuckerman et al. [56].

Unlike 2-oxoglutarate, ascorbate is not readily available in the human body. While most life forms can synthesize ascorbate from glucose, we must consume it as a part of our diet. From the stomach, the ascorbate is circulated in the blood stream and supplied all over the body. Estimated ascorbate uptake by the cells inside a microfluidic device, under similar extracellular concentrations available in plasma, is shown in figure 8(a), where experimental data for U937 cells [44] are also presented for comparison. It should be noted that the proposed parameters are based on the experimental data for first five hours of exposure to extracellular fluid. For longer exposures, the uptake starts to saturate and the parameters have to be modified accordingly.

For ascorbate transport in the microfluidic channel, a parabolic velocity profile with $200 \mu \mathrm{m} / \mathrm{s}$ mid-channel velocity was considered. Under hypoxic tension and ensuing reactions, the actual accumulation will vary from healthy cells. It should be mentioned that in oral administration of ascorbate, the plasma levels never exceed $220 \mu \mathrm{M}$ [74]. However, intravenous dosage on healthy human subjects has resulted in mean peak plasma concentrations of $885 \pm 201.2 \mu \mathrm{M}$ with a maximum tolerated upper limit of $13.4 \mathrm{mM}$ [42]. As our model suggests, intracellular levels will generally be much higher than plasma levels 

be toxic to cancer cells beyond $1 \mathrm{mM}$ of plasma levels $[42,75]$. This opens up the possibility of ascorbate as a target specific therapeutic agent for killing cancer cells through direct intravenous administration.

We studied four different concentrations of interest with the lower limit being close to the levels corresponding to an ascorbate-deficient diet to an upper limit showing a possible plasma level after intravenous administration (figure 8(b)) to illustrate the effect of ascorbate supplementation on the cell. Here, only the portion of the ascorbate accumulation due to the uptake process for a given extracellular concentration is presented in the vertical axis. The model predicts a very little increase in ascorbate accumulation when extracellular levels go beyond $1 \mathrm{mM}$. Model results also show that at much lower extracellular ascorbate case (for example $1 \mu \mathrm{M}$ ), cellular transmembrane ascorbate pumps remain highly active and this results in a much higher concentration of ascorbate inside the cell.

Here we particularly studied the effects of supplementing external ascorbate at $1 \%$ and $6 \%$ oxygen corresponding to severe and moderate levels of hypoxia. Since the interaction of ascorbate with the PFD is considered happening after the PFD is oxidized, for severe hypoxic conditions supplementation of ascorbate has little effect in reducing HIFmediated transcriptional activity (figure $9(a, b)$ ). This also suggests freely available ascorbate inside the cell that cannot participate in hydroxylating HIF. However, when a higher level of oxygen was available, it resulted in much more effective control of transcriptional response in all different ascorbate levels (figure $9(c, d)$ ). The apparent diminutive sensitivity of hypoxic response to variation in ascorbate levels in figure 9(b) and 9(d) can be attributed to the model choice of ascorbate independent hydroxylation [76]. However, given the wider response ascorbate has, even in severe hypoxic cases [77], we propose that high levels of ascorbate can bypass the oxygen dependence considered in our model which can be studied in greater detail in future with appropriate experimental measurements. 


\section{Conclusions}

A hybrid numerical model depicting cellular response in different hypoxic conditions has been presented to study the biophysical and biochemical behavior of cells in a microfluidic setup. Extracellular species transport is described using a modified unsteady Nernst-Planck equation, while the intracellular domain is compartmentalized between cytoplasm and nucleus where the temporal evolution of the species is resolved. We have developed an inhouse computer program to study a number of different cellular responses.

The model is used to predict the temporal extracellular oxygen concentration evolution in a hypoxia chamber. The numerically predicted results are in good agreement with experimental data suggesting that the model can be applied to predict extracellular species distribution under varying conditions. Moreover, a number of experimental results, both in normoxia and hypoxia, are used to validate the intracellular functions.

In the intracellular model, the species shuttling between cytosol and nucleus is introduced to investigate cell response in hypoxia in the microfluidic chip. Although we have studied the short time responses ( $\sim 1$ hour) of the cell, the present model can easily accommodate longer time responses. It should be noted that in a prolonged hypoxic scenario, cells can produce hypoxia-inducible factors in the nucleus in an autocrine fashion [58]. Moreover, ascorbate uptake dynamics over longer periods change due to intracellular saturation which should be addressed for temporal extension of the model beyond 5 hours [44]. Based on the results of the present model, the relative augmentation of hypoxia response inhibition using ascorbate supplementation was much lower than expected and reported in models exploring similar conditions [25]. This clearly indicates either a parallel PHD independent route for ascorbate-HIF interaction or an ascorbate concentration dependent change in PHD activity. Additionally, the asparaginyl hydroxylation pathway with factors inhibiting HIF (FIH) was not explicitly included in this present model. The major effect of this inhibition is the ablation of nuclear HIF binding to the transcriptional coactivator protein $\mathrm{p} 300 / \mathrm{CBP}$ [78]. In the present model, the transcriptional activation was lumped as a final step in the hypoxia response elements pathway in the nucleus. It is also worth mentioning that unlike PHD, FIH does not translocate to the nucleus with its HIF 
substrates [45]. The inclusion of these effects and parallel pathways in future models can bring the results of the model much closer to those of an in vitro microfluidic study mimicking cellular behavior in living tissue.

We used the model to study the effect of various extracellular and intracellular components in HIF hydroxylation pathway. Our study suggests a threshold for PHD concentration that can drastically affect hypoxia response. This type of control can potentially be applied as a therapeutic intervention. Another significantly important use of this kind of model lies in designing experiments in the microfluidic cell culture setup. Information from the model can serve as indicators of key parameters for study and provide valuable feedback in identifying potential factors for parametric variation studies, which can essentially produce a cost effective and efficient synergy of experiment and modeling.

6. Acknowledgment: This work was supported in part by the National Science Foundation under Grant No. DMS-1317671.

\section{References}

[1] D.M. Gilkes, G.L. Semenza, D. Wirtz, Hypoxia and the extracellular matrix: drivers of tumour metastasis, Nature Reviews Cancer, 14 (2014) 430-439.

[2] P. Vaupel, A. Mayer, M. Hockel, Tumor hypoxia and malignant progression, Oxygen Sensing, 381 (2004) 335-354.

[3] N.G. Machado, M.G. Alves, R.A. Carvalho, P.J. Oliveira, Mitochondrial Involvement in Cardiac Apoptosis During Ischemia and Reperfusion: Can We Close the Box?, Cardiovascular Toxicology, 9 (2009) 211-227.

[4] Y. Kominato, R. Iida, T. Nakajima, Y. Tajima, R. Takagi, C. Makita, K. Kishi, M. Ueki, Y. Kawai, T. Yasuda, Hypoxia induces upregulation of the deoxyribonuclease I gene in the human pancreatic cancer cell line QGP-1, Biochimica Et Biophysica Acta-General Subjects, 1770 (2007) 1567-1575.

[5] R.A. Gatenby, K. Smallbone, P.K. Maini, F. Rose, J. Averill, R.B. Nagle, L. Worrall, R.J. Gillies, Cellular adaptations to hypoxia and acidosis during somatic evolution of breast cancer, British Journal of Cancer, 97 (2007) 646-653. 
[6] O. Tredan, C.M. Galmarini, K. Patel, I.F. Tannock, Drug resistance and the solid tumor microenvironment, Journal of the National Cancer Institute, 99 (2007) 1441-1454.

[7] W.G. Kaelin, P.J. Ratcliffe, Oxygen sensing by metazoans: The central role of the HIF hydroxylase pathway, Molecular Cell, 30 (2008) 393-402.

[8] E. Berra, E. Benizri, A. Ginouves, V. Volmat, D. Roux, J. Pouyssegur, HIF prolyl-hydroxylase 2 is the key oxygen sensor setting low steady-state levels of HIF-1 alpha in normoxia, Embo Journal, 22 (2003) 4082-4090.

[9] M. Hirsila, P. Koivunen, V. Gunzler, K.I. Kivirikko, J. Myllyharju, Characterization of the human prolyl 4-hydroxylases that modify the hypoxia-inducible factor, Journal of Biological Chemistry, 278 (2003) 30772-30780.

[10] R. Depping, A. Steinhoff, S.G. Schindler, B. Friedrich, R. Fagerlund, E. Metzen, E. Hartmann, M. Koehler, Nuclear translocation of hypoxia-inducible factors (HIFs): Involvement of the classical importin alpha/beta pathway, Biochimica Et Biophysica Acta-Molecular Cell Research, 1783 (2008) 394-404.

[11] B. Keith, R.S. Johnson, M.C. Simon, HIF1 alpha and HIF2 alpha: sibling rivalry in hypoxic tumour growth and progression, Nature Reviews Cancer, 12 (2012) 9-22.

[12] V.H. Haase, Regulation of erythropoiesis by hypoxia-inducible factors, Blood Reviews, 27 (2013) 41-53.

[13] R. Kalluri, M. Zeisberg, Fibroblasts in cancer, Nature Reviews Cancer, 6 (2006) 392-401.

[14] A.A. Qutub, F. Mac Gabhann, E.D. Karagiannis, P. Vempati, A.S. Popel, Multiscale Models of Angiogenesis, Ieee Engineering in Medicine and Biology Magazine, 28 (2009) 14-31.

[15] G.L. Semenza, HIF-1 mediates metabolic responses to intratumoral hypoxia and oncogenic mutations, Journal of Clinical Investigation, 123 (2013) 3664-3671.

[16] K.S. Hewitson, C.J. Schofield, The HIF pathway as a therapeutic target, Drug Discovery Today, 9 (2004) 704-711.

[17] Z. Li, J. Wang, T. Zhou, X. Ye, Establishment of a colorectal cancer nude mouse visualization model of HIF-1 overexpression, Oncology Letters, 11 (2016) 2725-2732.

[18] M.L. Rexius-Hall, G. Mauleon, A.B. Malik, J. Rehman, D.T. Eddington, Microfluidic platform generates oxygen landscapes for localized hypoxic activation, Lab on a Chip, 14 (2014) 4688-4695.

[19] D. Pappas, Microfluidics and cancer analysis: cell separation, cell/tissue culture, cell mechanics, and integrated analysis systems, Analyst, 141 (2016) 525-535. 
[20] Y. Gao, P. Li, D. Pappas, A microfluidic localized, multiple cell culture array using vacuum actuated cell seeding: integrated anticancer drug testing, Biomedical Microdevices, 15 (2013) 907915.

[21] J. El-Ali, P.K. Sorger, K.F. Jensen, Cells on chips, Nature, 442 (2006) 403-411.

[22] G. Khanal, K. Chung, X. Solis-Wever, B. Johnson, D. Pappas, Ischemia/reperfusion injury of primary porcine cardiomyocytes in a low-shear microfluidic culture and analysis device, Analyst, 136 (2011) 3519-3526.

[23] Y. Kim, M.A. Stolarska, H.G. Othmer, The role of the microenvironment in tumor growth and invasion, Progress in Biophysics \& Molecular Biology, 106 (2011) 353-379.

[24] K.E. Sung, N. Yang, C. Pehlke, P.J. Keely, K.W. Eliceiri, A. Friedl, D.J. Beebe, Transition to invasion in breast cancer: a microfluidic in vitro model enables examination of spatial and temporal effects, Integrative Biology, 3 (2011) 439-450.

[25] A.A. Qutub, A.S. Popel, A computational model of intracellular oxygen sensing by hypoxiainducible factor HIF1 alpha, Journal of Cell Science, 119 (2006) 3467-3480.

[26] L.K. Nguyen, M.A.S. Cavadas, C.C. Scholz, S.F. Fitzpatrick, U. Bruning, E.P. Cummins, M.M. Tambuwala, M.C. Manresa, B.N. Kholodenko, C.T. Taylor, A. Cheong, A dynamic model of the hypoxia-inducible factor 1 alpha (HIF-1 alpha) network, Journal of Cell Science, 126 (2013) 14541463.

[27] Y. Kim, H.G. Othmer, A hybrid model of tumor-stromal interactions in breast cancer, Bulletin of Mathematical Biology, 75 (2013) 1304-1350.

[28] G. Khanal, S. Hiemstra, D. Pappas, Probing hypoxia-induced staurosporine resistance in prostate cancer cells with a microfluidic culture system, Analyst, 139 (2014) 3274-3280.

[29] M.D. Brennan, M.L. Rexius-Hall, L.J. Elgass, D.T. Eddington, Oxygen control with microfluidics, Lab on a Chip, 14 (2014) 4305-4318.

[30] J. Park, T. Bansal, M. Pinelis, M.M. Maharbiz, A microsystem for sensing and patterning oxidative microgradients during cell culture, Lab on a Chip, 6 (2006) 611-622.

[31] A.P. Vollmer, R.F. Probstein, R. Gilbert, T. Thorsen, Development of an integrated microfluidic platform for dynamic oxygen sensing and delivery in a flowing medium, Lab on a Chip, 5 (2005) 1059-1066.

[32] G.J. Cheng, D. Pirzada, P. Dutta, Design and fabrication of a hybrid nanofluidic channel, Journal of Microlithography Microfabrication and Microsystems, 4 (2005).

[33] T.K.J. Sze, J. Liu, P. Dutta, Numerical Modeling of Flow Through Phloem Considering Active Loading, Journal of Fluids Engineering-Transactions of the Asme, 136 (2014). 
[34] M.C. Kim, R.H.W. Lam, T. Thorsen, H.H. Asada, Mathematical analysis of oxygen transfer through polydimethylsiloxane membrane between double layers of cell culture channel and gas chamber in microfluidic oxygenator, Microfluidics and Nanofluidics, 15 (2013) 285-296.

[35] E.L. Cussler, Diffusion: Mass Transfer in Fluid Systems, Cambridge University Press2009.

[36] P.M. Herst, M.V. Berridge, Cell surface oxygen consumption: A major contributor to cellular oxygen consumption in glycolytic cancer cell lines, Biochimica Et Biophysica Acta-Bioenergetics, 1767 (2007) 170-177.

[37] R.A. Gatenby, R.J. Gillies, Why do cancers have high aerobic glycolysis?, Nature Reviews Cancer, 4 (2004) 891-899.

[38] B.A. Wagner, S. Venkataraman, G.R. Buettner, The rate of oxygen utilization by cells, Free Radical Biology and Medicine, 51 (2011) 700-712.

[39] J. Du, J.J. Cullen, G.R. Buettner, Ascorbic acid: Chemistry, biology and the treatment of cancer, Biochimica Et Biophysica Acta-Reviews on Cancer, 1826 (2012) 443-457.

[40] X.M. Wu, T. Iguchi, J. Hirano, I. Fujita, H. Ueda, N. Itoh, K. Tanaka, T. Nakanishi, Upregulation of sodium-dependent vitamin $\mathrm{C}$ transporter 2 expression in adrenals increases norepinephrine production and aggravates hyperlipidemia in mice with streptozotocin-induced diabetes, Biochemical Pharmacology, 74 (2007) 1020-1028.

[41] J.F. Graumlich, T.M. Ludden, C. ConryCantilena, L.R. Cantilena, Y.H. Wang, M. Levine, Pharmacokinetic model of ascorbic acid in healthy male volunteers during depletion and repletion, Pharmaceutical Research, 14 (1997) 1133-1139.

[42] S.J. Padayatty, H. Sun, Y.H. Wang, H.D. Riordan, S.M. Hewitt, A. Katz, R.A. Wesley, M. Levine, Vitamin C pharmacokinetics: Implications for oral and intravenous use, Annals of Internal Medicine, 140 (2004) 533-537.

[43] C. Malo, J.X. Wilson, Glucose modulates vitamin C transport in adult human small intestinal brush border membrane vesicles, Journal of Nutrition, 130 (2000) 63-69.

[44] J.M. Vislisel, F.Q. Schafer, G.R. Buettner, A simple and sensitive assay for ascorbate using a plate reader, Analytical Biochemistry, 365 (2007) 31-39.

[45] R. Depping, W. Jelkmann, F.K. Kosyna, Nuclear-cytoplasmatic shuttling of proteins in control of cellular oxygen sensing, Journal of Molecular Medicine-Jmm, 93 (2015) 599-608.

[46] E. Metzen, U. Berchner-Pfannschmidt, P. Stengel, J.H. Marxsen, I. Stolze, M. Klinger, W.Q. Huang, C. Wotzlaw, T. Hellwig-Burgel, W. Jelkmann, H. Acker, J. Fandrey, Intracellular localisation of human HIF-1 alpha hydroxylases: implications for oxygen sensing, Journal of Cell Science, 116 (2003) $1319-1326$. 
[47] A.C.R. Epstein, J.M. Gleadle, L.A. McNeill, K.S. Hewitson, J. O'Rourke, D.R. Mole, M. Mukherji, E. Metzen, M.I. Wilson, A. Dhanda, Y.M. Tian, N. Masson, D.L. Hamilton, P. Jaakkola, R. Barstead, J. Hodgkin, P.H. Maxwell, C.W. Pugh, C.J. Schofield, P.J. Ratcliffe, C-elegans EGL-9 and mammalian homologs define a family of dioxygenases that regulate HIF by prolyl hydroxylation, Cell, 107 (2001) 43-54.

[48] N. Goda, S.J. Dozier, R.S. Johnson, HIF-1 in cell cycle regulation, apoptosis, and tumor progression, Antioxidants \& Redox Signaling, 5 (2003) 467-473.

[49] R. Myllylä, L. Tuderman, K.I. Kivirikko, Mechanism of the prolyl hydroxylase reaction, European Journal of Biochemistry, 80 (1977) 349-357.

[50] M. Hirsilä, Characterization of the novel human prolyl 4-hydroxylases and asparaginyl hydroxylase that modify the hypoxia-inducible factor, Department of Medical Biochemistry and Molecular Biology, University of Oulu, Finland, 2004, pp. 99.

[51] T. Kamura, D.M. Koepp, M.N. Conrad, D. Skowyra, R.J. Moreland, O. Iliopoulos, W.S. Lane, W.G. Kaelin, S.J. Elledge, R.C. Conaway, J.W. Harper, J.W. Conway, Rbx1, a component of the VHL tumor suppressor complex and SCF ubiquitin ligase, Science, 284 (1999) 657-661.

[52] K. Yoo, J. Shim, J. Liu, P. Dutta, Mathematical and numerical model to study two-dimensional free flow isoelectric focusing, Biomicrofluidics, 8 (2014).

[53] I.B. Sprague, P. Dutta, Improved kinetics from ion advection through overlapping electric double layers in nano-porous electrodes, Electrochimica Acta, 91 (2013) 20-29.

[54] J. Shim, P. Dutta, C.F. Ivory, Finite-volume methods for isotachophoretic separation in microchannels, Numerical Heat Transfer Part a-Applications, 52 (2007) 441-461.

[55] S. Patankar, Numerical heat transfer and fluid flow, CRC press1980.

[56] J.R. Tuckerman, Y.G. Zhao, K.S. Hewitson, Y.M. Tian, C.W. Pugh, P.J. Ratcliffe, D.R. Mole, Determination and comparison of specific activity of the HIF-prolyl hydroxylases, Febs Letters, 576 (2004) 145-150.

[57] E.E. Spaeth, S.K. Friedlander, The diffusion of oxygen, carbon dioxide, and inert gas in flowing blood, Biophys J, 7 (1967) 827-851.

[58] A.A. Qutub, A.S. Popel, Three autocrine feedback loops determine HIF1 alpha expression in chronic hypoxia, Biochimica Et Biophysica Acta-Molecular Cell Research, 1773 (2007) 1511-1525.

[59] D.P. Stiehl, R. Wirthner, J. Koditz, P. Spielmann, G. Camenisch, R.H. Wenger, Increased prolyl 4hydroxylase domain proteins compensate for decreased oxygen levels - Evidence for an autoregulatory oxygen-sensing system, Journal of Biological Chemistry, 281 (2006) 23482-23491. 

Imamura, Hexokinase II and VEGF expression in liver tumors: correlation with hypoxia-inducible factor-1 alpha and its significance, Journal of Hepatology, 40 (2004) 117-123.

[61] L. Gossage, T. Eisen, E.R. Maher, VHL, the story of a tumour suppressor gene, Nature Reviews Cancer, 15 (2015) 55-64.

[62] T. Shuin, K. Kondo, S. Kaneko, N. Sakai, M. Yao, M. Hosaka, H. Kanno, S. Ito, I. Yamamoto, Results of mutation analyses of von Hippel-Lindau disease gene in Japanese patients: Comparison with results in United States and United Kingdom, Acta Urologica Japonica, 41 (1995) 703-707.

[63] L. Gossage, M. Murtaza, A.F. Slatter, C.P. Lichtenstein, A. Warren, B. Haynes, F. Marass, I. Roberts, S.J. Shanahan, A. Claas, A. Dunham, A.P. May, N. Rosenfeld, T. Forshew, T. Eisen, Clinical and pathological impact of VHL, PBRM1, BAP1, SETD2, KDM6A, and JARID1c in clear cell renal cell carcinoma, Genes Chromosomes \& Cancer, 53 (2014) 38-51.

[64] C.L. Cioffi, X.Q. Liu, P.A. Kosinski, M. Garay, B.R. Bowen, Differential regulation of HIF-1 alpha prolyl-4-hydroxylase genes by hypoxia in human cardiovascular cells, Biochemical and Biophysical Research Communications, 303 (2003) 947-953.

[65] J.H. Marxsen, P. Stengel, K. Doege, P. Heikkinen, T. Jokilehto, T. Wagner, W. Jelkmann, P. Jaakkola, E. Metzen, Hypoxia-inducible factor-1 (HIF-1) promotes its degradation by induction of HIF-alpha-prolyl-4-hydroxylases, Biochemical Journal, 381 (2004) 761-767.

[66] L.A. McNeill, E. Flashman, M.R.G. Buck, K.S. Hewitson, I.J. Clifton, G. Jeschke, T.D.W. Claridge, D. Ehrismann, N.J. Oldham, C.J. Schofield, Hypoxia-inducible factor prolyl hydroxylase 2 has a high affinity for ferrous iron and 2-oxoglutarate, Molecular Biosystems, 1 (2005) 321-324.

[67] R. Fukuda, H. Zhang, J.-w. Kim, L. Shimoda, C.V. Dang, G.L. Semenza, HIF-1 regulates cytochrome oxidase subunits to optimize efficiency of respiration in hypoxic cells, Cell, 129 (2007) 111-122.

[68] J. Aragones, M. Schneider, K. Van Geyte, P. Fraisl, T. Dresselaers, M. Mazzone, R. Dirkx, S. Zacchigna, H. Lemieux, N.H. Jeoung, D. Lambrechts, T. Bishop, P. Lafuste, A. Diez-Juan, S.K. Harten, P. Van Noten, K. De Bock, C. Willam, M. Tjwa, A. Grosfeld, R. Navet, L. Moons, T. Vandendriessche, C. Deroose, B. Wijeyekoon, J. Nuyts, B. Jordan, R. Silasi-Mansat, F. Lupu, M. Dewerchin, C. Pugh, P. Salmon, L. Mortelmans, B. Gallez, F. Gorus, J. Buyse, F. Sluse, R.A. Harris, E. Gnaiger, P. Hespel, P. Van Hecke, F. Schuit, P. Van Veldhoven, P. Ratcliffe, M. Baes, P. Maxwell, P. Carmeliet, Deficiency or inhibition of oxygen sensor Phd1 induces hypoxia tolerance by reprogramming basal metabolism, Nature Genetics, 40 (2008) 170-180.

[69] W.M. Bernhardt, V. Campean, S. Kany, J.S. Jurgensen, A. Weidemann, C. Warnecke, M. Arend, S. Klaus, V. Gunzler, K. Amann, C. Willam, M.S. Wiesener, K.U. Eckardt, Preconditional activation of 
hypoxia-inducible factors ameliorates ischemic acute renal failure, Journal of the American Society of Nephrology, 17 (2006) 1970-1978.

[70] R.R. Ratan, A. Siddiq, N. Smirnova, K. Karpisheva, R. Haskew-Layton, S. McConoughey, B. Langley, A. Estevez, P.T. Huerta, B. Volpe, S. Roy, C.K. Sen, I. Gazaryan, S. Cho, M. Fink, J. LaManna, Harnessing hypoxic adaptation to prevent, treat, and repair stroke, Journal of Molecular MedicineJmm, 85 (2007) 1331-1338.

[71] R.V. Shohet, J.A. Garcia, Keeping the engine primed: HIF factors as key regulators of cardiac metabolism and angiogenesis during ischemia, Journal of Molecular Medicine-Jmm, 85 (2007) 1309-1315.

[72] M.A. Selak, S.M. Armour, E.D. MacKenzie, H. Boulahbel, D.G. Watson, K.D. Mansfield, Y. Pan, M.C. Simon, C.B. Thompson, E. Gottlieb, Succinate links TCA cycle dysfunction to oncogenesis by inhibiting HIF-alpha prolyl hydroxylase, Cancer Cell, 7 (2005) 77-85.

[73] G.L. Semenza, Hypoxia-inducible factor 1: Regulator of mitochondrial metabolism and mediator of ischemic preconditioning, Biochimica Et Biophysica Acta-Molecular Cell Research, 1813 (2011) 1263-1268.

[74] S.J. Padayatty, H.D. Riordan, S.M. Hewitt, A. Katz, L.J. Hoffer, M. Levine, Intravenously administered vitamin C as cancer therapy: three cases, Canadian Medical Association Journal, 174 (2006) 937-942.

[75] M.V. Clement, J. Ramalingam, L.H. Long, B. Halliwell, The in vitro cytotoxicity of ascorbate depends on the culture medium used to perform the assay and involves hydrogen peroxide, Antioxidants \&amp; Redox Signaling, 3 (2001) 157-163.

[76] L. Tuderman, R. Myllylä, K.I. Kivirikko, Mechanism of the prolyl hydroxylase reaction, European Journal of Biochemistry, 80 (1977) 341-348.

[77] C. Kuiper, I.G.M. Molenaar, G.U. Dachs, M.J. Currie, P.H. Sykes, M.C.M. Vissers, Low ascorbate levels are associated with increased hypoxia-inducible factor-1 activity and an aggressive tumor phenotype in endometrial cancer, Cancer Research, 70 (2010) 5749-5758.

[78] D. Lando, D.J. Peet, D.A. Whelan, J.J. Gorman, M.L. Whitelaw, Asparagine hydroxylation of the HIF transactivation domain: A hypoxic switch, Science, 295 (2002) 858-861.

[79] J.M. Berg, J.L. Tymoczko, L. Stryer, Biochemistry, W.H. Freeman2002.

[80] B.H. Jiang, G.L. Semenza, C. Bauer, H.H. Marti, Hypoxia-inducible factor 1 levels vary exponentially over a physiologically relevant range of 0-2 tension, American Journal of PhysiologyCell Physiology, 271 (1996) C1172-C1180. 
[81] U.R. Jewell, I. Kvietikova, A. Scheid, C. Bauer, R.H. Wenger, M. Gassmann, Induction of HIF-1 alpha in response to hypoxia is instantaneous, Faseb Journal, 15 (2001) 1312-1314.

[82] E. Berra, D. Roux, D.E. Richard, J. Pouyssegur, Hypoxia-inducible factor-1 alpha (HIF-1 alpha) escapes 0-2-driven proteasomal degradation irrespective of its subcellular localization: nucleus or cytoplasm, Embo Reports, 2 (2001) 615-620. 


\section{List of Tables}

Table 1: Initial and boundary conditions for transport equations in a rectangular shape microfluidic extracellular domain. The length (x-direction) of the computation domain is $5.5 \mathrm{~cm}$ and the width (y-direction) of the computation domain is 300 microns. Since a qusi2D model is used in this study, the boundary contribution in the height (z) direction is taken care in the governing equations.

\begin{tabular}{|l|l|l|l|l|}
\hline & Initial conditions $^{\mathrm{a}}$ & Inlet $^{\mathrm{b}}(\mathrm{x}=0)$ & $\begin{array}{l}\text { Side } \text { walls }^{\mathrm{c}} \quad(\mathrm{y}= \pm 150 \\
\mu \mathrm{m})\end{array}$ & Outlet $^{\mathrm{d}}(\mathrm{x}=5.5 \mathrm{~cm})$ \\
\hline Oxygen & $C_{O}(x, y)=207 \mu M$ & $C_{O}=C_{\text {given }}$ & $\frac{\partial C_{O}}{\partial y}=0$ & $\frac{\partial C_{O}}{\partial x}=0$ \\
\hline Ascorbate & $C_{A S}(x, y)=1 m M$ & $C_{A S}=C_{\text {given }}$ & $\frac{\partial C_{A S}}{\partial y}=0$ & $\frac{\partial C_{A S}}{\partial x}=0$ \\
\hline
\end{tabular}

a Unless otherwise mentioned

b Dirichlet conditions chosen according to species supply concentration. Default concentrations, reported in table $\mathrm{S} 3$, are used unless otherwise mentioned

c The side walls are assumed impermeable to the nutrients

$\mathrm{d}$ The concentrations are lengthwise invariant and the flow is fully developed at the outlet 
Table 2: Parameters required for the calculation of cellular oxygen consumption and ascorbate uptake

\begin{tabular}{|c|c|c|}
\hline Parameter & Value & Reference \\
\hline$\rho_{\text {cell }}$ & $3.33 \mathrm{e}+10$ cells $/ \mathrm{m}^{3}$ & \multirow{2}{*}{ Calculated $^{1}$} \\
\hline$k_{m, O}$ & $1.0 \mu \mathrm{M}$ & \\
\hline$V_{\max , O, U 937}$ & $11.0 \mathrm{e}-18 \mathrm{~mol} /$ (cell.s) \\
\hline$V_{\max , O, H e L a}$ & $12.5 \mathrm{e}-18 \mathrm{~mol} /$ (cell.s) & \\
\hline$V_{\max , A S}$ & $4.188 \mathrm{e}-7 \mathrm{~mol} /($ cell.s) & Calculated $^{2}$ \\
\hline$k_{m, A S}$ & $63.698 \mu \mathrm{M}$ & Calculated $^{2}$ \\
\hline
\end{tabular}

${ }^{1}$ The cell density was calculated by dividing the number of cells considered by the total volume of the channel.

2 A double-reciprocal (Lineweaver-Burk) was used to calculate the Michaelis-Menten parameters for ascorbate uptake [79]. Experimental results [44], which presented a direct linear increase (0-5 hours) of intracellular ascorbate as a function of extracellular concentrations $(50 \mu \mathrm{M}-1 \mathrm{mM})$, were used for the calculations. The rate of uptake at each concentration was calculated from data points in the linear range. Based on the rate and the corresponding extracellular ascorbate concentration, the Lineweaver-Burk plot was constructed, and the intercepts with the horizontal and vertical axes gave the inverse of the Michaelis-Menten parameters $k_{m, A S}$ and $V_{\max , A S}$. 
Table 3: Species considered in the model and their symbols

\begin{tabular}{|c|c|}
\hline Notation & Description \\
\hline$O$ & Oxygen \\
\hline$A S$ & Ascorbate \\
\hline$P H D$ & Prolyl Hydroxylases \\
\hline$F e$ & Iron \\
\hline$P F$ & Binding of Iron and Prolyl Hydroxylases \\
\hline$D G$ & Binding of PF and 2-oxoglutarate \\
\hline$P F D$ & Binding of PFD and Oxygen \\
\hline$P F D O$ & Binding of PFDO and Ascorbate \\
\hline$P F D O A$ & Hypoxia-inducible factor 1 $\alpha$ \\
\hline$H I F 1 \alpha$ & PFDOA and HIF intermediate \\
\hline$P F D A \cdot H I F 1 \alpha$ & Hypoxia-inducible factor 1 $\alpha$, hydroxylated \\
\hline$H I F 1 \alpha h$ & Hypoxia-inducible factor 1 $\beta$ \\
\hline$H I F 1 \beta$ & Dimer of HIF1 $\alpha$ and HIF1 $\beta$ \\
\hline$H I F_{d}$ & Hypoxia response elements \\
\hline$H R E$ & Binding of HIFd and HRE \\
\hline$H I F_{d} H R E$ & von Hippel-Lindau \\
\hline$v H L$ & Quantity inside nucleus \\
\hline$T A$ &
\end{tabular}




\section{List of Figures}

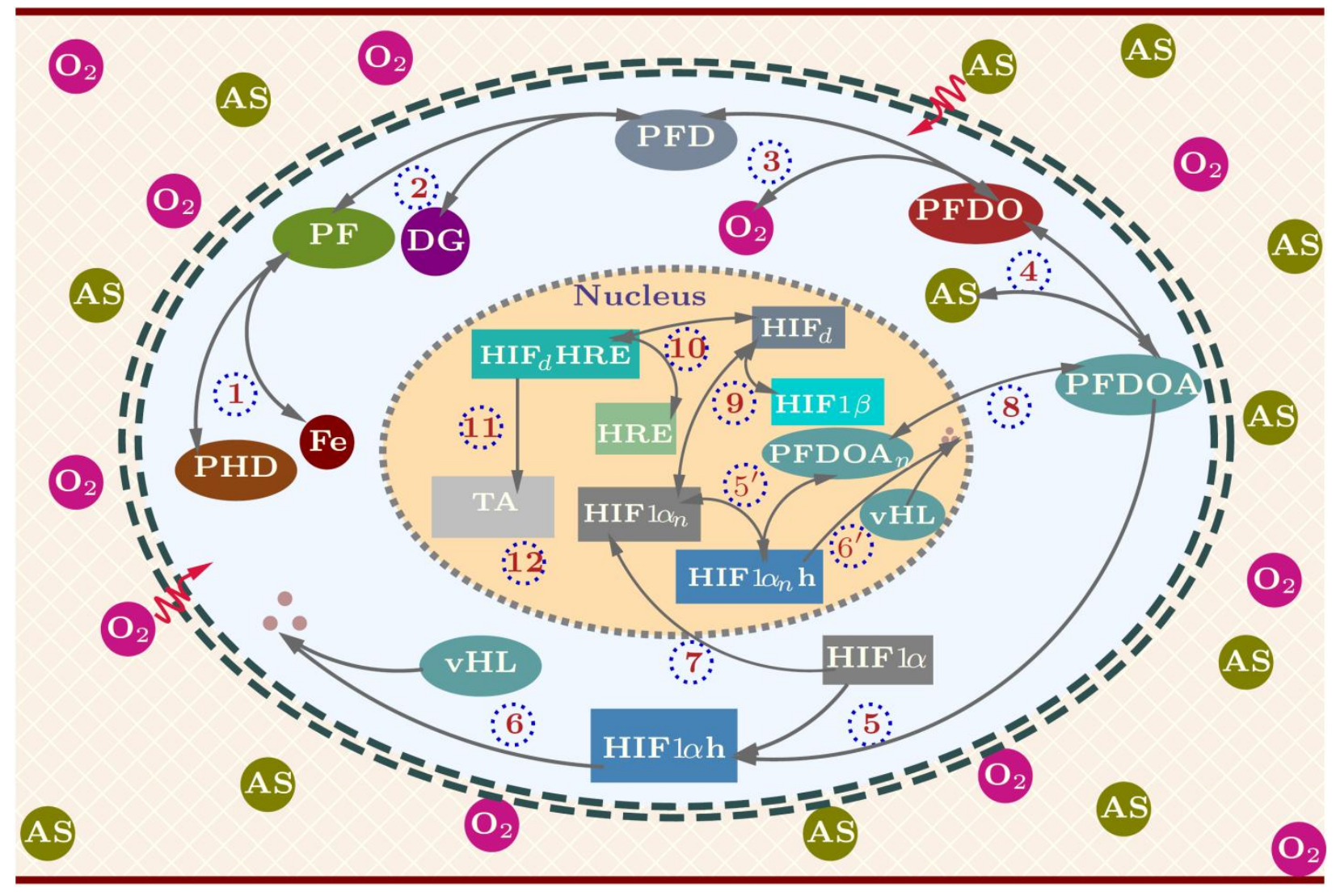

Fig. 1. Schematic of the normoxic and hypoxic reaction pathways. Under normoxic conditions prolyl hydroxylase domains (PHD) form complex with iron (Fe), 2-oxoglutarate (DG), oxygen $\left(\mathrm{O}_{2}\right)$ and ascorbate (AS) (1-2-3-4), and hydroxylates the hypoxia-inducible factors (HIF1 $\alpha$ ) (5), which later gets degraded by ubiquitination reaction after being tagged by von Hippel-Lindau (vHL) proteins (6). Under oxygen tension, more HIF1 $\alpha$ is available to pass inside the nucleus (7), where aside from being engaged by PHD complex (5'-6'), they undergo dimerization with HIF1 $\beta$ (9) which is primarily available inside the nucleus. The dimer (HIFd) then reacts with hypoxia response elements (HRE), and eventually serves as the key element in transcriptionally activating (TA) a wide array of genes responsible for angiogenesis, tumor progression, and survival. Shuttling of PHD in and out of the nucleus (8) has been assumed to occur after catalytic activations (1-2-3-4) for simplicity, and uptake of extracellular nutrients $\mathrm{CO}_{2}$ and AS) has been considered for proper representation of the biophysical activities. 

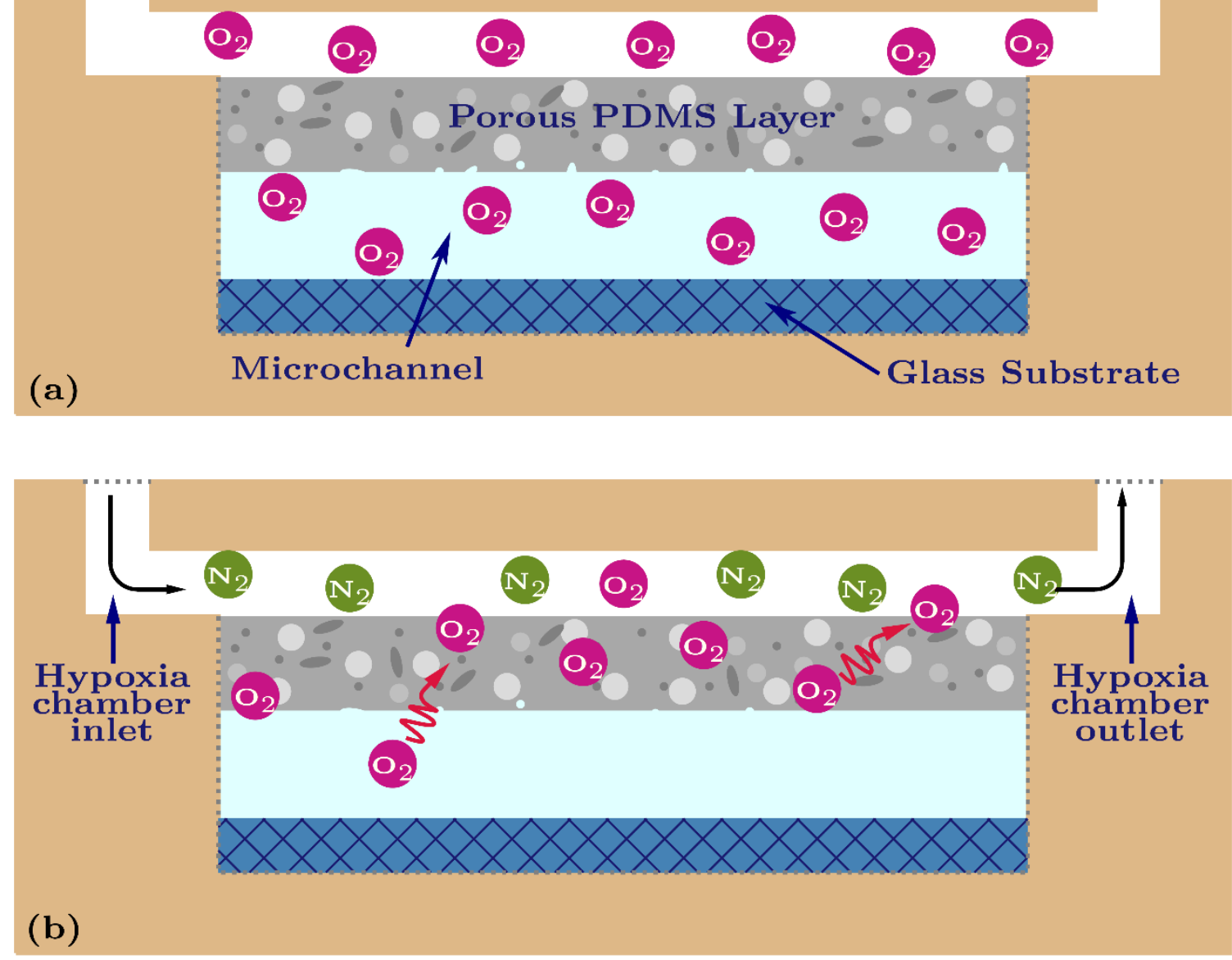

Fig. 2. Schematic representation of the oxygen control mechanism in hypoxia chamber used in the model following the experimental setup in [28] with minor variation for model formulation. (a) The microfluidic chip containing cell culture is placed inside the hypoxia chamber. Ambient air over the chip maintains oxygen balance in the setup; (b) As the air over the chip is flushed with nitrogen, the sharp difference is oxygen level across the porous PDMS layer of the chip induces permeation of oxygen from the microchannel to the environment outside. This results in hypoxic states inside the chip. The oxygen level outside can be tuned as needed to maintain a required hypoxic level inside the chip. 


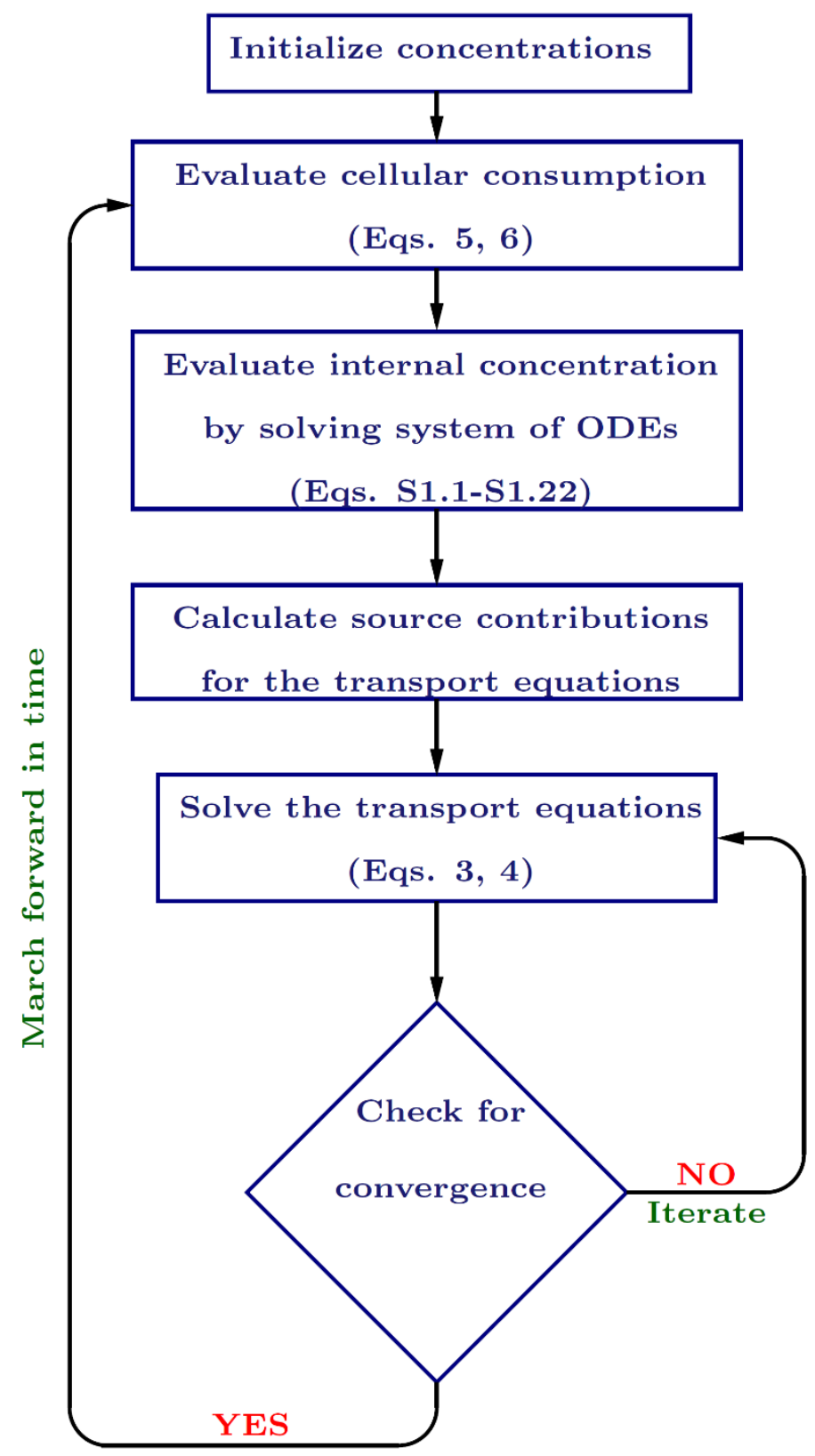

Fig. 3. Flowchart for the hybrid algorithm designed to solve the coupled temporal intracellular and the spatiotemporal extracellular domain. Since the extracellular concentration changes due to cellular consumption and uptake from inside the cell, and due to convection-diffusion and permeation outside, it is necessary to evaluate extracellular nutrient levels at each time-step. This also directly affects the intracellular consumption and uptake thus creating a coupled system that is solved by iterations. 


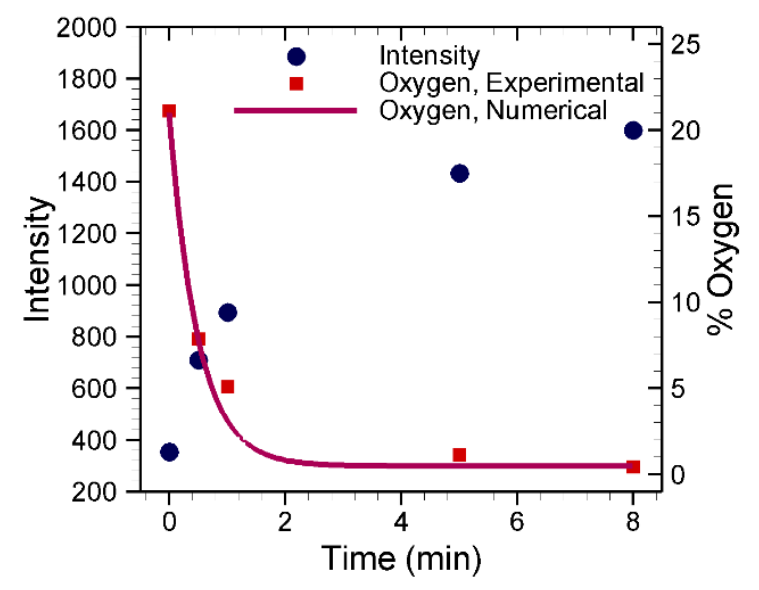

Fig. 4. Model results emulating the induction of hypoxia inside a microfluidic device. Outside the microfluidic chip, there was no oxygen due to nitrogen flushing. Inside the chip, the model started with normoxic conditions ( $21 \%$ oxygen). Numerical solution over the eight-minute periods, inside the hypoxia chamber, is shown as the solid line. Experimental fluorescence intensity of oxygen marker is shown as blue circles [28]. Lower oxygen levels during hypoxia result in reduced fluorescence quenching and higher intensity (shown as red squares). 

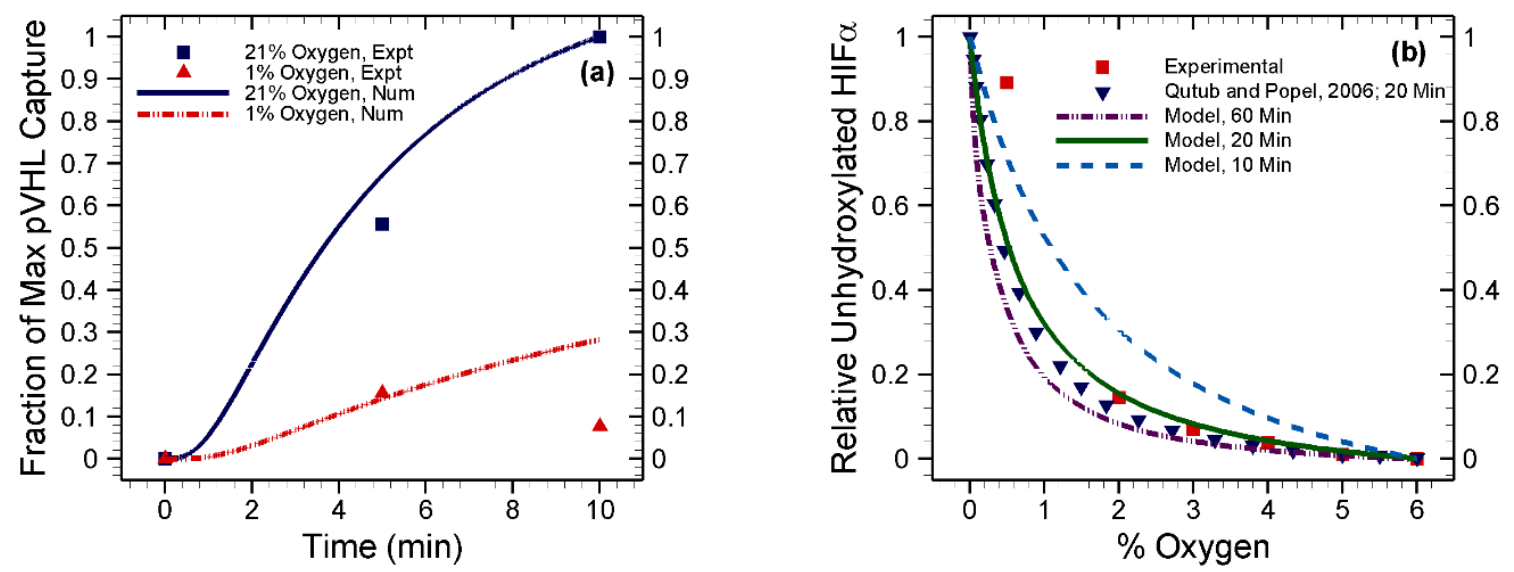

Fig. 5. Cellular HIF1 $\alpha$ dynamics in normoxia and hypoxia. (a) vHL capture of hydroxylated HIF1 $\alpha$ in normoxic $(21 \%)$ and hypoxic (1\%) oxygen levels. Experimental results from [56] with HEK293T cells are used for comparison with model predictions where the initial PHD concentration is $4 \mathrm{nM}$. PHD-modified HIF $1 \alpha \mathrm{h}$ is quickly captured by $\mathrm{vHL}$ protein ligase, but the amount of hydroxylation is a strong function of oxygen concentration. (b) Model predictions of relative unhydroxylated HIF $1 \alpha$ at different oxygen tensions ranging from $0 \%$ to $6 \%$ with experimental results from [80]. Both the model and experimental data are normalized to the level obtained at $6 \%$ oxygen. Under hypoxic conditions, the amount of HIF $1 \alpha$ available increases steeply with a drop in oxygen concentration. Initial conditions have been reported in table S3. 

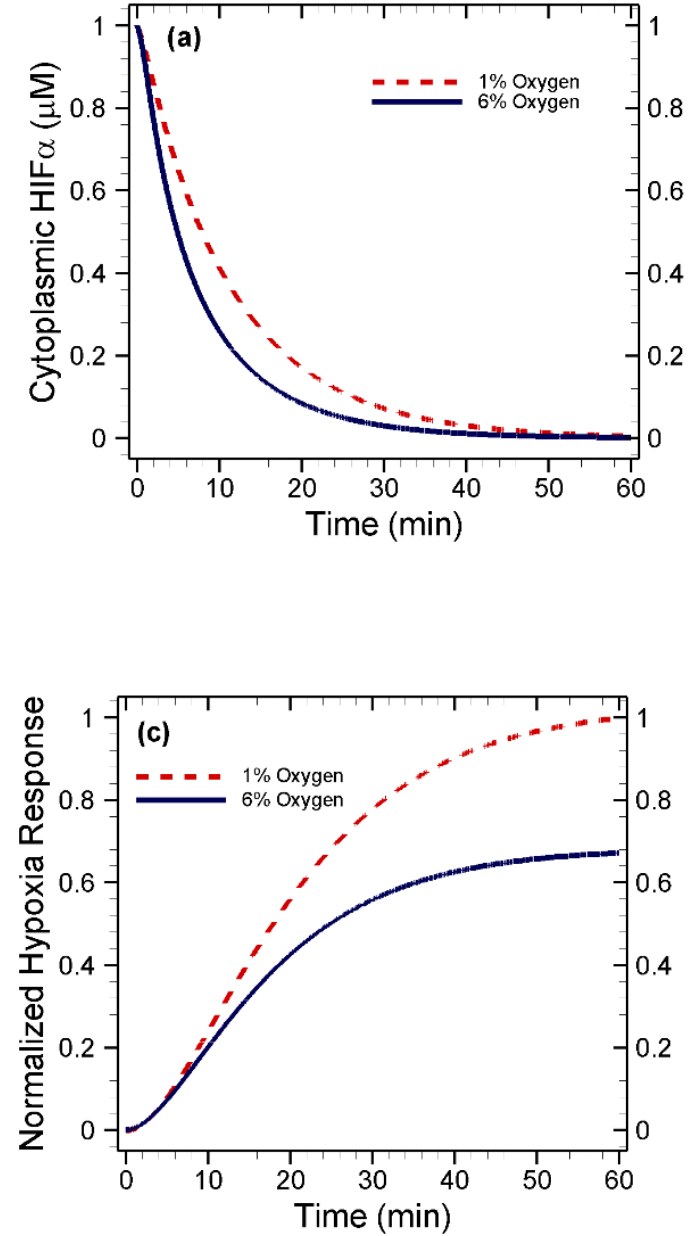
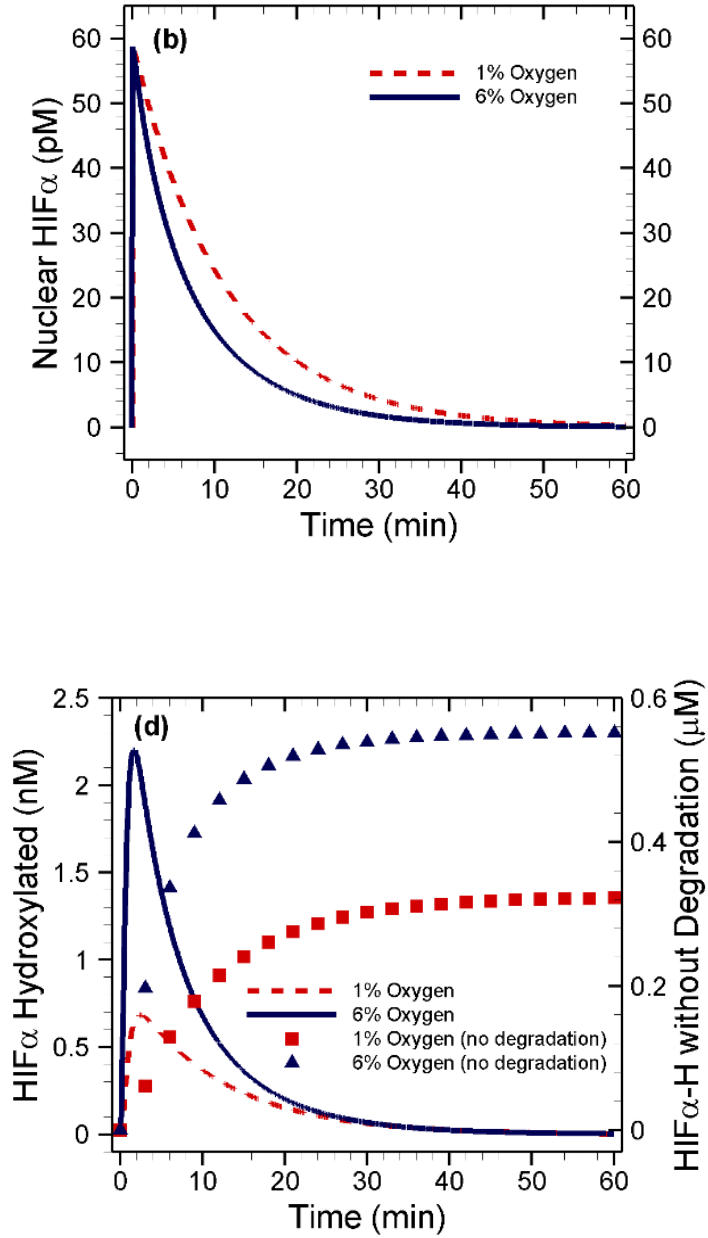

Fig. 6. Hypoxic response under varying degrees of hypoxia over one hour. The initial concentration of all species in the PHD-HIF pathway are reported in table S3. (a) Model prediction of unhydroxylated cytoplasmic HIF $1 \alpha$ levels in $1 \%$ and $6 \%$ oxygen over one hour. Drop in cytoplasmic HIF1 $\alpha$ is due to nuclear shuttling as well as hydroxylation, (b) Evolution of nuclear HIF $1 \alpha$ in $1 \%$ and $6 \%$ oxygen. (c) Normalized transcriptional activity inside the nucleus resulting from HIF $1 \alpha$ shuttling in hypoxia. Transcriptional responses were normalized with respect to the maximum level attained after 60 minutes in 1\% oxygen. (d) Comparison of hydroxylated HIF1 $\alpha$ accumulation in presence and absence of vHL degradation. After hydroxylation, HIF1 $\alpha$ is degraded quickly by vHL. Right axis is showing the total HIF1 $\alpha$ hydroxylated over time considering no proteasomal degradation. This amount also includes the small portion of HIF $1 \alpha \mathrm{h}$ inside the nucleus. 

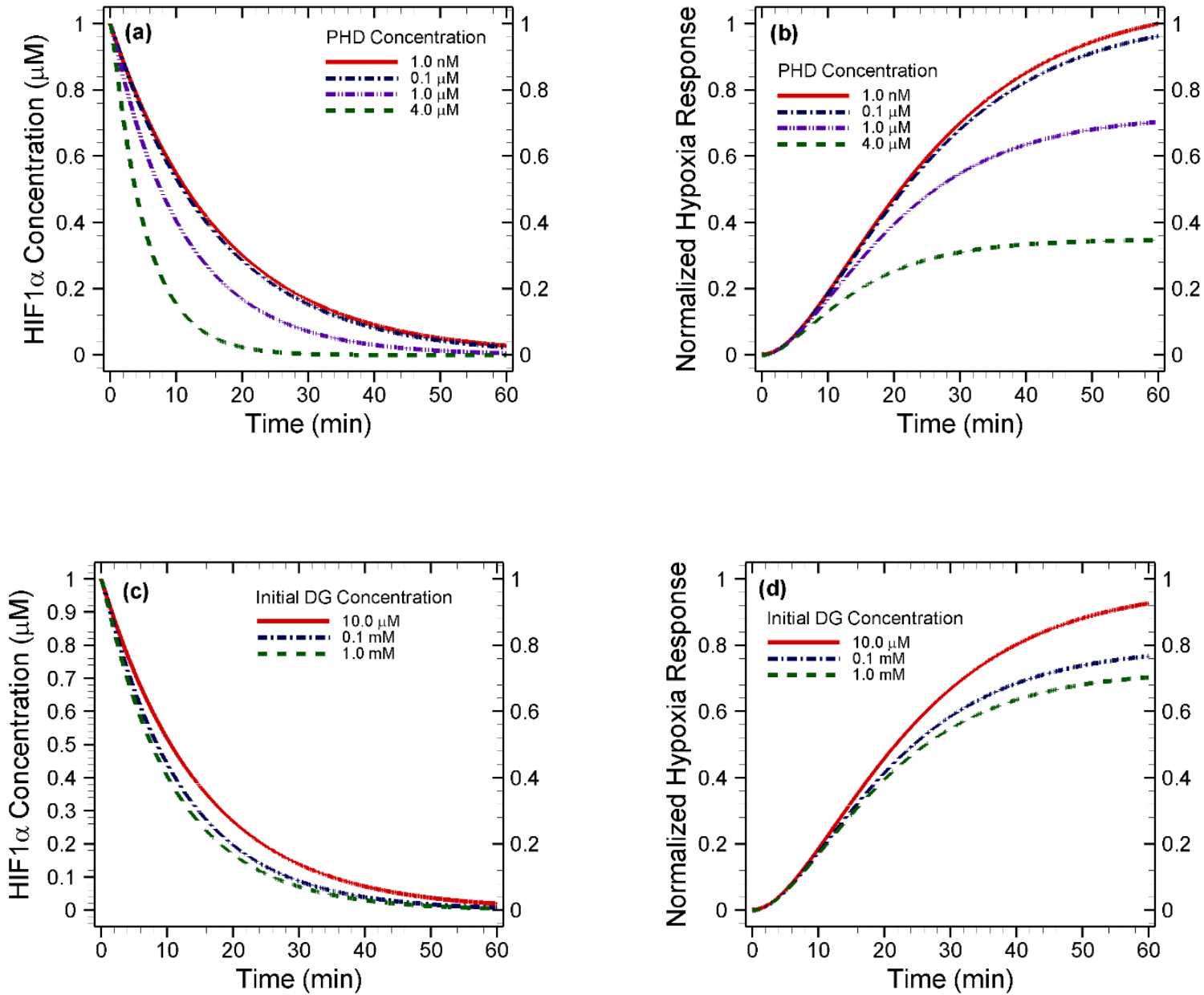

Fig. 7. Model results highlighting the relative effect of supplementing/limiting PHD and 2 -oxoglutarate on the hypoxic response (1\% Oxygen). Hypoxia response levels for all cases are normalized by the maximum response found in the case of $1.0 \mathrm{nM}$ PHD at the end of one hour. (a) Variation of cytosolic HIF1 $\alpha$ level in 60 minutes with different levels of PHD. Increasing PHD levels four orders from $1.0 \mathrm{nM}$ to $10 \mu \mathrm{M}$ results in a transition of HIF dynamics from a gradual to switch-like drop, (b) Change of normalized transcriptional activity in response to changing PHD levels over the one hour period. (c) Cytosolic HIF1 $\alpha$ levels with varying 2-oxoglutarate in the system from $10 \mu \mathrm{M}$ to $1 \mathrm{mM}$. (d) Normalized hypoxia response highlighting the effect of increasing oxoglutarate levels during a period of 60 minutes. This increase results in a significant drop in transcriptional activity over the range. All initial conditions except the species under variation are maintained as reported in table S3. 

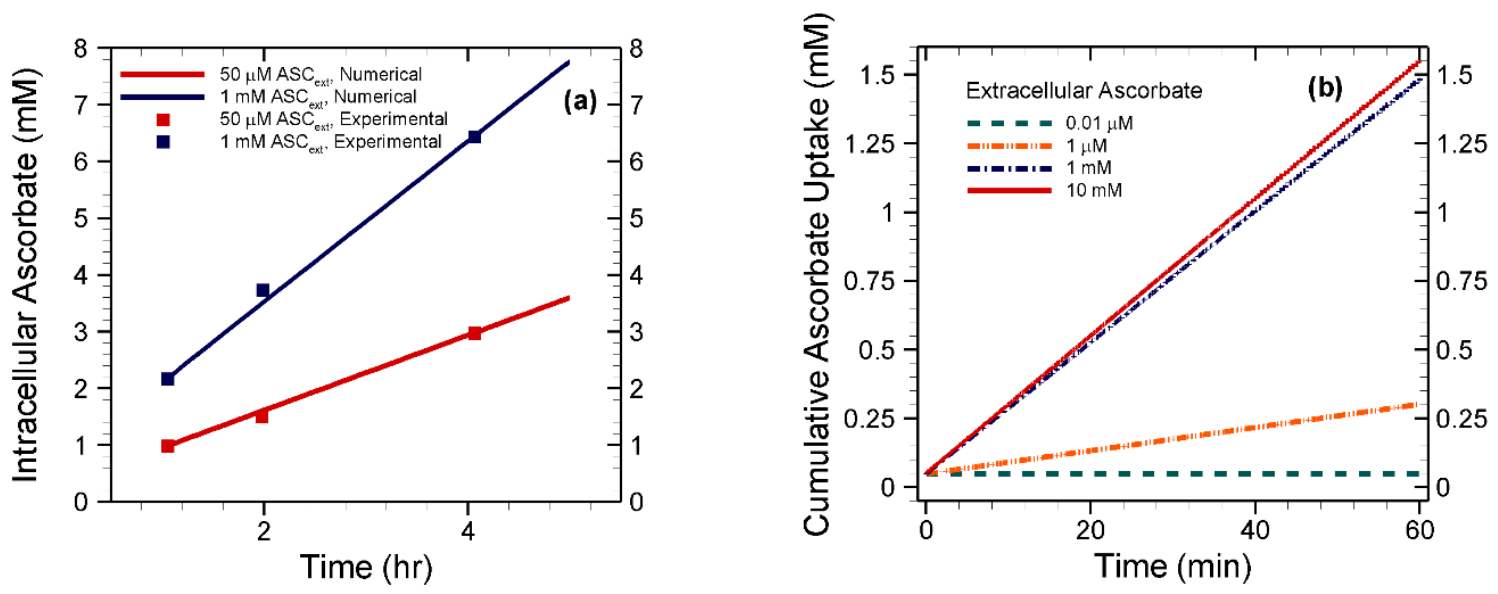

Fig. 8. Cellular ascorbate uptake model. (a) Model prediction of intracellular ascorbate accumulation with time in normoxia. Two experimental plasma ascorbate levels of $50 \mu \mathrm{M}$ and $1 \mathrm{mM}$ are considered as extracellular concentrations. Only the solution of cellular uptake over 5 hours is plotted and compared with experimental data from [44]. (b) Model prediction of cumulative ascorbate uptake by transporter proteins level over 60 minutes inside the microfluidic cell culture device. Four different extracellular supplementation levels from $0.01 \mu \mathrm{M}$ to $10 \mathrm{mM}$ in the extracellular medium was considered. Implementation of the uptake model in hypoxic reaction system $(1 \%$ oxygen) results in high accumulation of intracellular ascorbate over 60 minutes even for as low as $1 \mu \mathrm{M}$ plasma ascorbate concentration. 

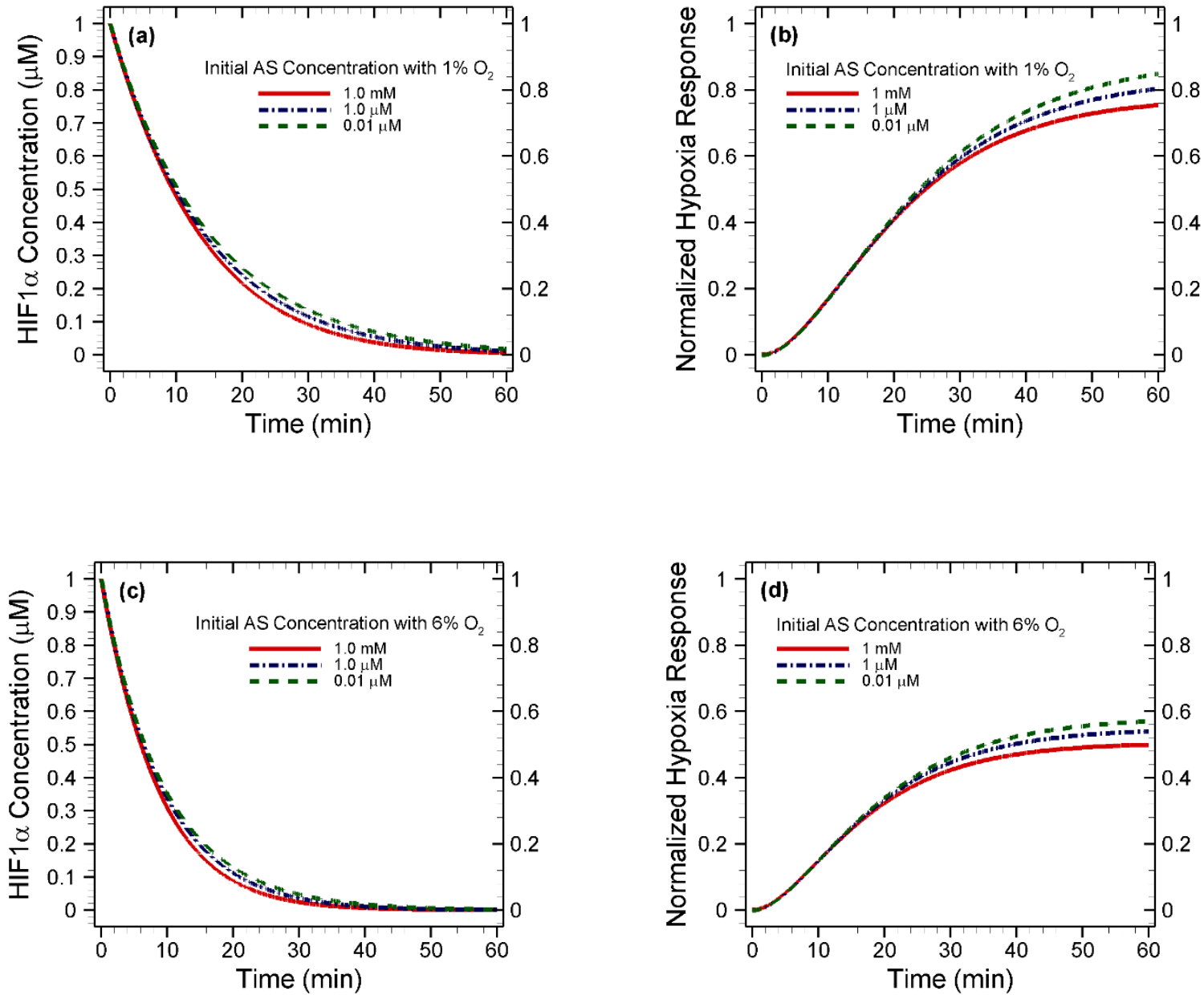

Fig. 9. Model results highlighting the relative effect of ascorbate supplementation at different hypoxic conditions. (a) Cytosolic HIF1 $\alpha$ concentration with ascorbate levels of $0.01 \mu \mathrm{M}, 1.0 \mu \mathrm{M}$, and $1.0 \mathrm{mM}$ at $1 \%$ oxygen, (b) HIF1 $\alpha$ transcriptional activity with varying ascorbate levels at $1 \%$ oxygen. (c) HIF $1 \alpha$ concentration with different levels of ascorbate supplementation at $6 \%$ oxygen. A slight increase (from $1 \%$ to $6 \%$ oxygen changes) HIF1 $\alpha$ hydroxylation dynamics showing a move towards switch-like response and (d) HIF1 $\alpha$ transcriptional activity with varying ascorbate levels at $6 \%$ oxygen. Increase in upstream oxygen results in noticeably reduced transcriptional activity compared to $1 \%$ oxygen. Transcriptional activity levels for figure 9 (b) and (d) are normalized by the maximum response found in the lowest level of PHD (Fig. 7(b)). All initial conditions except ascorbate and oxygen are maintained as reported in table S3. 